\title{
可见光催化炔烃串联氰基烷基磺酰化/环化
}

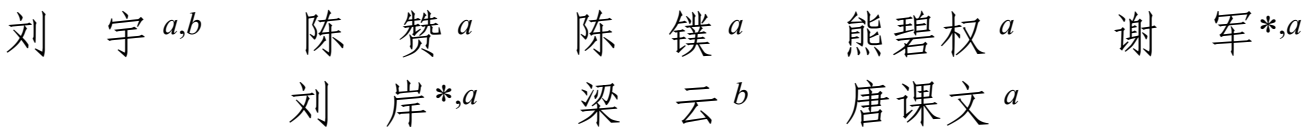

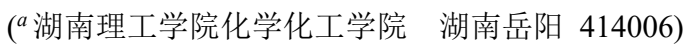 \\ $(b$ 湖南师范大学 湖南省有机功能分子组装与应用重点实验室 长沙 410081)
}

\begin{abstract}
摘要 报道了一种在无过渡金属条件下可见光催化炔烃与环酮肜衍生物的串联氧基烷基磺酰化/环化反应，通过 $\mathrm{SO}_{2}$ 的 插入合成 2-氰基烷基磺酰基- $9 H$-吡咯并 [1,2- $a$ ] 吲哚. 该碳碳参键的双官能团化包括自由基机理, 依次经历了亚胺自由 基的形成、环酮的开环、 $\mathrm{SO}_{2}$ 的插入、磺酰基对碳碳参键的加成、分子内环化和异构化.

关键词 可见光催化; $\mathrm{SO}_{2}$ 插入; 环酮肜酯; $N$-炔丙基吲哚
\end{abstract}

\section{Visible-Light-Catalyzed Tandem Cyanoalkylsulfonylation/ Cyclization of Alkynes}

\author{
Liu, $\mathrm{Yu}^{a, b} \quad$ Chen, $\mathrm{Zan}^{a} \quad \mathrm{Chen}^{\mathrm{P}} \mathrm{Pu}^{a} \quad \mathrm{Xiong}$ Biquan $^{a} \quad \mathrm{Xie}, \mathrm{Jun}^{*, a}$ \\ Liu, An ${ }^{*, a}$ Liang, Yun ${ }^{b} \quad$ Tang, Kewen ${ }^{a}$ \\ ( ${ }^{a}$ Department of Chemistry and Chemical Engineering, Hunan Institute of Science and Technology, Yueyang, Hunan 414006) \\ $\left({ }^{b}\right.$ Key Laboratory of the Assembly and Application of Organic Functional Molecules of Hunan Province, \\ Hunan Normal University, Changsha 410081)
}

\begin{abstract}
A transition-metal-free visible-light-mediated tandem cyanoalkylsulfonylation/cyclization of alkynes with cycloketone oxime derivatives for the construction of 2-cyanoalkylsulfonyl-9H-pyrrolo[1,2-a]indoles through the insertion of $\mathrm{SO}_{2}$ is reported. The difunctionalization of carbon-carbon triple bonds includes a radical mechanism and undergoes the formation of iminyl radical, ring-opening of cycloketone, insertion of $\mathrm{SO}_{2}$, addition of sulfonyl radical to carbon-carbon triple bonds, intramolecular cyclization and isomerization.

Keywords visible-light-catalysis; the insertion of $\mathrm{SO}_{2}$; cycloketone oxime esters; $\mathrm{N}$-propargylindoles
\end{abstract}

\section{Introduction}

Nitriles, especially alkylnitriles, widely presented in bioactive molecules and natural products, are important synthetic intermediates in organic chemistry and pharmaceutical industry, agriculture, material and polymer science. ${ }^{[1]}$ Many methods have been established for the construction of alkylnitrile derivatives. Among them, the most efficient and green strategy is functionalization of $\alpha-\mathrm{C}\left(\mathrm{sp}^{3}\right)-\mathrm{H}$ bonds in alkylnitriles. ${ }^{[2-4]}$ Recently, the radical ring-opening of cyclobutanone oximes has become a convenient and efficient route to introduce long-chain cyanoalkyl groups into organic molecules. ${ }^{[5-8]}$

Sulfones, a typical organosulfur compounds, are widely used in agrochemicals, chemical industry, organic synthesis, pharmaceuticals and antiflaming materials. ${ }^{[10-11]}$ Thus, many scientists are devoted to the development of simple and convenient methods for the construction of sulfonecontaining compounds. ${ }^{[12-13]}$ Among these methods, the insertion of $\mathrm{SO}_{2}$, which used DABSO $\left[\mathrm{DABCO} \cdot\left(\mathrm{SO}_{2}\right)_{2}\right],{ }^{[14]}$ thiourea dioxide ${ }^{[15]}$ and sodium sulphites ${ }^{[16]}$ as $\mathrm{SO}_{2}$ sources, is the most convenient and green strategy for the preparation of sulfones.

In 1991, Zard and co-workers ${ }^{[5]}$ reported the first

\footnotetext{
* Corresponding authors. E-mail: xiejun12018014@163.com; 352905634@qq.com

Received February 27, 2021; revised March 18, 2021; published online March 25, 2021.

Project supported by the National Natural Science Foundation of China (Nos. 22078084, 51874132), the Scientific Research Fund of Hunan Provincial Education Department (Nos. 20A224, 20A213, 18B355), the Science and Technology Planning Project of Hunan Province (Nos. 2020RC3056, 2018TP1017) and the Natural Science Foundation of Hunan Province (Nos. 2020JJ5221, 2020JJ5212).

国家自然科学基金(Nos. 22078084, 51874132)、湖南省教育厅科学研究基金(Nos. 20A224, 20A213, 18B355)、湖南省科技计划项目(Nos. 2020RC3056, 2018TP1017)、湖南省自然科学基金(No. 2020JJ5221, 2020JJ5212)资助项目.
} 
radical ring-opening of cyclobutanone carboxymethyl oximes or sulphenylimines to synthesize alkylnitriles. Subsequently, Uemura, ${ }^{[5 b-5 c]}$ Selander, ${ }^{[5 d-5 e]}$ Xiao and Chen, ${ }^{[6 \mathrm{a}-6 \mathrm{e}]} \mathrm{Guo},{ }^{[7 \mathrm{a}-7 \mathrm{~d}]} \mathrm{Yu},{ }^{[7 \mathrm{e}]} \mathrm{Wu},{ }^{[7 \mathrm{f}]} \mathrm{Li}$ 's $\operatorname{groups}^{[7 \mathrm{~g}]}$ and other groups ${ }^{[8]}$ have also developed the similar cleavage of carbon-carbon bond in cyclobutanone oximes. In recent years, several cyanoalkyl-sulfonylation reactions via ring-opening of cycloketone oxime derivatives and $\mathrm{SO}_{2}$ insertion have been developed. ${ }^{[8 \mathrm{a}-8 \mathrm{~d}]}$ Our group ${ }^{[8 \mathrm{a}]}$ reported Fe-mediated cyanoalkylsulfonylation of acrylamides with cycloketone oxime derivatives via $\mathrm{SO}_{2}$ insertion (path I, Scheme 1). Wu et al. ${ }^{[8 \mathrm{~b}]}$ disclosed visible-light-catalyzed cyanoalkylsulfonylation/alkoxylation of alkenes by using $O$-acyl oximes for trapping $\mathrm{SO}_{2}$ (path II, Scheme 1). Our group $^{[8 c]}$ also developed visible-light-mediated dual carbon-carbon bond cleavage method for the construction of 2-cyanoalkylsulfonyl-3,4-dihydronaphthalenes through $\mathrm{SO}_{2}$ insertion (path III, Scheme 1). In 2019, Lu's group ${ }^{[8 \mathrm{~d}]}$ reported photoredox-catalyzed $\mathrm{SO}_{2}$-insertion reaction by using cyclobutanone oxime derivatives as alkyl radical precursors for trapping $\mathrm{SO}_{2}$ (path IV, Scheme 1). These cyanoalkylsulfonylation reactions utilized cyanoalkyl radicals, which generated from nitrogen radical directed ${ }^{[9]}$ ring-opening of cycloketone oxime derivatives, to capture $\mathrm{SO}_{2}$. However, all of them employed carbon-carbon double bonds as cyanoalkyl radical acceptors. The strategy for cyanoalkylsulfonylation of carbon-carbon triple bonds is lacking.

The alkynes, which used in many radical trapping reactions, were common radical acceptors. ${ }^{[17]}$ The $N$-propargylindoles could capture carbon-radicals, ${ }^{[17 \mathrm{a}]}$ sulfur-radicals $^{[17 \mathrm{~b}-17 \mathrm{~g}]}$ or phosphorus-radicals ${ }^{[17 \mathrm{~h}-17 \mathrm{i}]}$ to afford 3-func- tionalized 9H-pyrrolo[1,2- $a]$ indoles. Recently, visiblelight-catalysis, which is an efficient and convenient strategy, is widely utilized in drug synthesis and organic synthesis due to its mild and simple conditions, safety, availability and high efficiency. ${ }^{[18-20]}$ Thus, we report a transition-metal-free visible-light-mediated tandem cyanoalkylsulfonylation/cyclization of alkynes to prepare 2cyanoalkylsulfonyl-9H-pyrrolo[1,2-a]indoles from $\mathrm{N}$-propargylindoles and cycloketone oxime derivatives via the insertion of $\mathrm{SO}_{2}$, in which two carbon-sulfur bonds and one carbon-carbon bond are constructed (Scheme 2).

\section{Results and discussion}

To gain the best reaction conditions, we began to investigate the reaction between 1-(3-phenylprop-2-yn-1yl)- $1 H$-indole (1a, $0.2 \mathrm{mmol})$ with cyclobutylidenemethyl 4-(trifluoromethyl)benzoate (2a, $0.3 \mathrm{mmol}, 1.5$ equiv.) in the presence of $\mathrm{Na}_{2}$-Eosin $\mathrm{Y}(2.8 \mathrm{mg}, 0.004 \mathrm{mmol}, 2$ mol\%), $\mathrm{K}_{2} \mathrm{~S}_{2} \mathrm{O}_{5}$ (88.9 mg, $0.4 \mathrm{mmol}, 2$ equiv.), $\mathrm{CH}_{3} \mathrm{CN}$ (2 $\mathrm{mL}$ ) as solvent at $80{ }^{\circ} \mathrm{C}$ under $5 \mathrm{~W}$ blue LED irradiation for $16 \mathrm{~h}$ (Table 1). To our surprise, the product 3aa could be isolated in $81 \%$ yield (Entry 1 ). Initially, several cyclobutanone $O$-acyloximes $\mathbf{2 a - 1} \sim \mathbf{2 a - 6}$ were examined (Entries 2 7). It was found that structural modification of the acyl moiety on the oxime had remarkable effect on the reaction. All of them could afford the sulfonylation products and none of them could afford higher yield than that of cyclobutanone oxime $\mathbf{2 a}$. Then, other sulfur dioxide sources, including DABSO, thiourea dioxide and $\mathrm{Na}_{2} \mathrm{~S}_{2} \mathrm{O}_{5}$, were investigated. $\mathrm{K}_{2} \mathrm{~S}_{2} \mathrm{O}_{5}$ was the best sulfur dioxide source according to the reaction yields (Entries $8 \sim 10$ ).

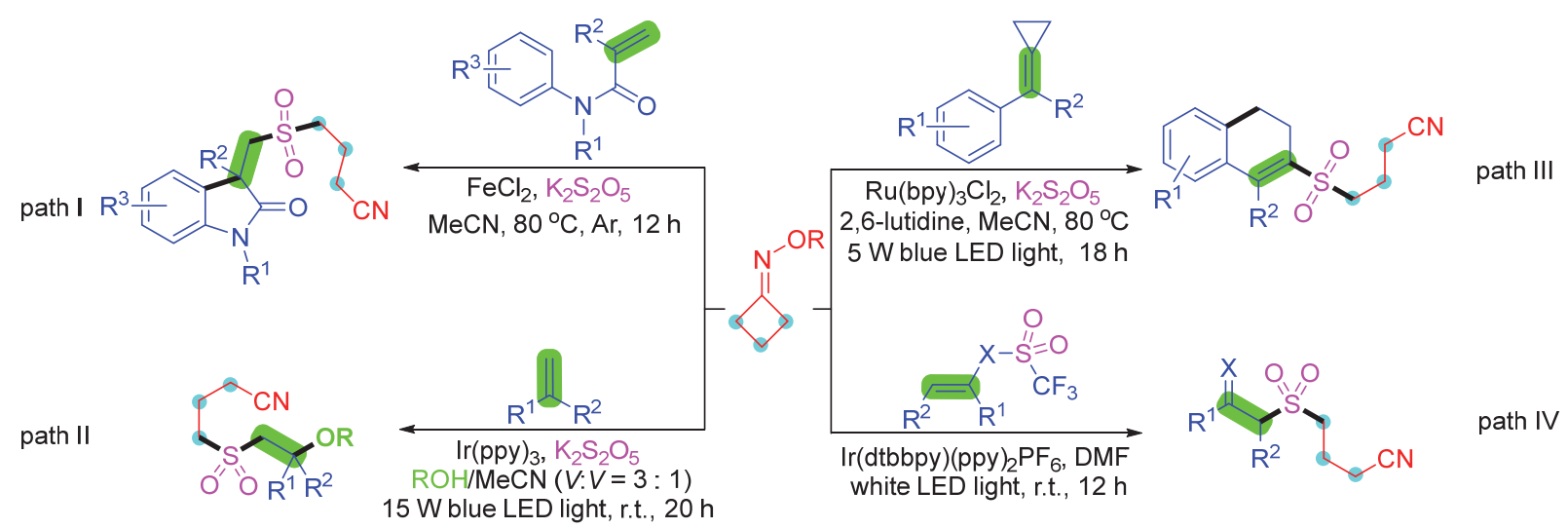

Scheme 1 In situ $\mathrm{SO}_{2}$-capture transformations of cycloketone oxime esters

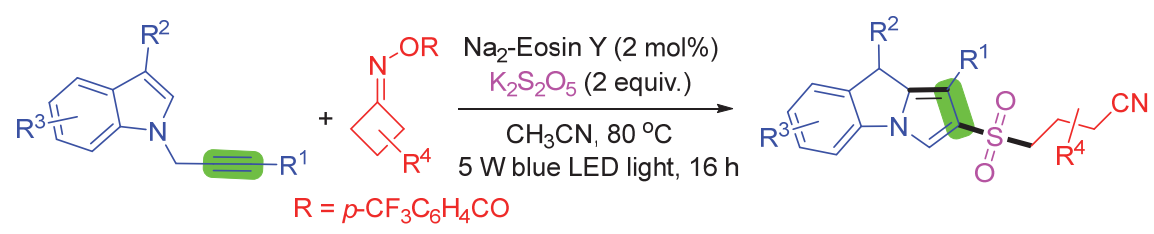

Scheme 2 Tandem cyanoalkylsulfonylation/cyclization of alkynes via $\mathrm{SO}_{2}$ insertion 
Table 1 Screening optimal conditions ${ }^{a}$

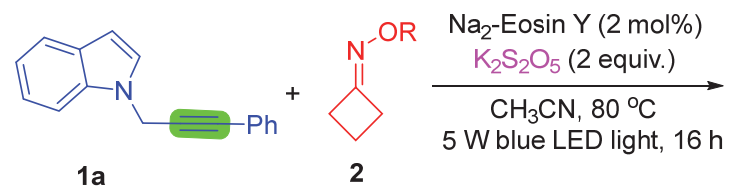

2a, $\mathrm{R}=p-\mathrm{CF}_{3} \mathrm{C}_{6} \mathrm{H}_{4} \mathrm{CO} ; \mathbf{2 a}-\mathbf{1}, \mathrm{R}=\mathrm{CF}_{3} \mathrm{CO}$; 2a-2, R = $\mathrm{C}_{6} \mathrm{H}_{5} \mathrm{CO} ; 2 \mathrm{a}-3, \mathrm{R}=0-\mathrm{NO}_{2} \mathrm{C}_{6} \mathrm{H}_{4} \mathrm{CO}$; $2 \mathrm{a}-4, \mathrm{R}=p-\mathrm{NO}_{2} \mathrm{C}_{6} \mathrm{H}_{4} \mathrm{CO} ; \mathbf{2} \mathrm{a}-5, \mathrm{R}=p-\mathrm{FC}_{6} \mathrm{H}_{4} \mathrm{CO}$ 2a-6, $\mathrm{R}=\mathrm{C}_{6} \mathrm{~F}_{5} \mathrm{CO}$

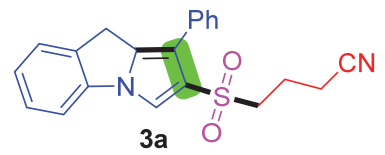

\begin{tabular}{|c|c|c|}
\hline Entry & Variation from the standard conditions & Yield/\% \\
\hline 1 & None & 81 \\
\hline 2 & $\mathbf{2 a - 1}$ instead of $\mathbf{2 a}$ & 13 \\
\hline 3 & $\mathbf{2 a - 2}$ instead of $\mathbf{2 a}$ & 25 \\
\hline 4 & $\mathbf{2 a - 3}$ instead of $\mathbf{2 a}$ & 46 \\
\hline 5 & 2a-4 instead of $\mathbf{2 a}$ & 55 \\
\hline 6 & $\mathbf{2 a - 5}$ instead of $\mathbf{2 a}$ & 51 \\
\hline 7 & $\mathbf{2 a - 6}$ instead of $\mathbf{2 a}$ & 50 \\
\hline 8 & DABSO instead of $\mathrm{K}_{2} \mathrm{~S}_{2} \mathrm{O}_{5}$ & 8 \\
\hline 9 & Thiourea dioxide instead of $\mathrm{K}_{2} \mathrm{~S}_{2} \mathrm{O}_{5}$ & 67 \\
\hline $10^{c}$ & $\mathrm{Na}_{2} \mathrm{~S}_{2} \mathrm{O}_{5}$ instead of $\mathrm{K}_{2} \mathrm{~S}_{2} \mathrm{O}_{5}$ & 73 \\
\hline $11^{c}$ & Without $\mathrm{Na}_{2}$-Eosin $\mathrm{Y}$ & 0 \\
\hline 12 & $\operatorname{Ir}(p p y)_{3}$ instead of $\mathrm{Na}_{2}-\mathrm{E}$ osin $\mathrm{Y}$ & 59 \\
\hline 13 & $\mathrm{Ru}(\mathrm{bpy})_{3} \mathrm{Cl}_{2}$ instead of $\mathrm{Na}_{2}$-Eosin $\mathrm{Y}$ & 34 \\
\hline 14 & Eosin $\mathrm{Y}$ instead of $\mathrm{Na}_{2}$-Eosin $\mathrm{Y}$ & 64 \\
\hline 15 & Without additional light & 0 \\
\hline 16 & $\mathrm{Na}_{2}$-Eosin $\mathrm{Y}(5 \mathrm{~mol} \%)$ & 81 \\
\hline 17 & Toluene instead of $\mathrm{CH}_{3} \mathrm{CN}$ & 40 \\
\hline 18 & THF instead of $\mathrm{CH}_{3} \mathrm{CN}$ & 38 \\
\hline $19^{c}$ & DMF instead of $\mathrm{CH}_{3} \mathrm{CN}$ & 27 \\
\hline $20^{c}$ & Dioxane instead of $\mathrm{CH}_{3} \mathrm{CN}$ & 44 \\
\hline 21 & DMSO instead of $\mathrm{CH}_{3} \mathrm{CN}$ & 32 \\
\hline 22 & $3 \mathrm{~W}$ blue LED light & 61 \\
\hline 23 & $12 \mathrm{~W}$ blue LED light & 54 \\
\hline 24 & $36 \mathrm{~W}$ compact fluorescent light & 45 \\
\hline 25 & At $100{ }^{\circ} \mathrm{C}$ & 70 \\
\hline 26 & At $60{ }^{\circ} \mathrm{C}$ & 42 \\
\hline 27 & For $32 \mathrm{~h}$ & 80 \\
\hline $28^{d}$ & None & 52 \\
\hline
\end{tabular}

${ }^{a}$ Reaction conditions: 1a $(0.2 \mathrm{mmol}), 2 \mathrm{a}(0.3 \mathrm{mmol}, 1.5$ equiv. $), \mathrm{K}_{2} \mathrm{~S}_{2} \mathrm{O}_{5}(88.9$ $\mathrm{mg}, 0.4 \mathrm{mmol}, 2$ equiv.), $\mathrm{Na}_{2}$-Eosin $\mathrm{Y}(2.8 \mathrm{mg}, 2 \mathrm{~mol} \%), \mathrm{CH}_{3} \mathrm{CN}(2 \mathrm{~mL})$ at $80{ }^{\circ} \mathrm{C}$ and $5 \mathrm{~W}$ blue LED irradiation for $16 \mathrm{~h} .{ }^{b}$ Isolated yield. ${ }^{c}$ The rest of substrate 1a was recovered. ${ }^{d} \mathbf{1 a}(1.0 \mathrm{~g}, 4.33 \mathrm{mmol}), \mathbf{2 a}$ (1.5 equiv.), $\mathrm{Na}_{2}$-Eosin $\mathrm{Y}(2 \mathrm{~mol} \%), \mathrm{K}_{2} \mathrm{~S}_{2} \mathrm{O}_{5}$ (2 equiv.) and $\mathrm{MeCN}(40 \mathrm{~mL})$ at $80{ }^{\circ} \mathrm{C}$ for $64 \mathrm{~h}$.

Conducting the reaction without photocatalyst could not give the target product (Entry 11). Other phtotocatalysts were screened. The results showed that $\mathrm{Na}_{2}$-Eosin $\mathrm{Y}$ were suitable (Entries 12 14). Conducting the reaction without additional light could not give the target product (Entry 15). These results indicated that the visible-light and photocatalyst were indispensable for the reaction. Then, Increasing the amount of $\mathrm{Na}_{2}$-Eosin $\mathrm{Y}$ to $5 \mathrm{~mol} \%$ could still not increase the reaction yield (Entry 16). Subsequently, several kinds of other solvents, including toluene, tetrahydrofuran (THF), $N, N$-dimethylformamide (DMF), dioxane and dimethyl sulfoxide (DMSO) were tested. None of them afforded higher yields than that of $\mathrm{CH}_{3} \mathrm{CN}$ (Entries 17 21). The investigation of different light sources suggested that $5 \mathrm{~W}$ blue LED was the best choice (Entries 22 24). Increasing the temperature to $100{ }^{\circ} \mathrm{C}$ or decreasing the temperature to $60{ }^{\circ} \mathrm{C}$ did not give satisfactory reaction yields (Entries $25 \sim 26$ ). Prolonging the reaction time to 32 $\mathrm{h}$ could not increase the reaction yield obviously (Entry 27). A gram scale experiment could produce the product 3 aa in moderate yield (Entry 28).

According to the acquired best conditions, we set out to study the scope of $N$-propargylindoles 1 and $O$-acyl oximes 2 (Table 2). First, a variety of substituted $N$-propargylindoles $\mathbf{1 b} \sim \mathbf{1 s}$ bearing different substituents at the terminal of $\mathrm{C}-\mathrm{C}$ triple bonds were examined. $\mathrm{N}$-propargylindoles $\mathbf{1 b} \sim \mathbf{1 p}$ bearing different aromatic ring at the terminal of alkynes could undergo the tandem cyclization smoothly and generated the target corresponding products 3ba $\sim 3$ pa in $54 \% \sim 85 \%$ yields. The yields of target products were affected by both electronic effect and steric effect of the substituents. The electron-donating groups substituted substrates gave higher yields than electronwithdrawing groups substituted ones. The ortho- and metasubstituted group substrates give lower yields than para-substituted ones. The $N$-propargylindoles $\mathbf{1 q}\left(\mathrm{R}^{1}=\right.$ thienyl) and $\mathbf{1 r}\left(\mathrm{R}^{1}=\mathrm{Me}\right)$ could assemble the target products 3qa and 3ra in moderate yields. Nevertheless, 1-(prop-2-yn-1-yl)- $1 H$-indole (1s) $\quad\left(\mathrm{R}^{1}=\mathrm{H}\right)$ was not suitable for the transformation (product 3sa). Subsequently, the $N$-propargylindoles $1 \mathbf{1 t} \sim \mathbf{1 a a}$ bearing substituents on the indole skeleton were tested (products 3ta $\sim \mathbf{3 a a a}$ ). All of these substrates $\mathbf{1 t} \sim \mathbf{1 a a}$ were good candidates for this reaction and delivered the corresponding products in good yields. It is worth noting that the halo-substituted substrates were compatible with the standard conditions. The halo-substituted positions provided a chance for further modification. Next, different cycloketone oximes $\mathbf{2 b} \sim \mathbf{2 n}$, including 2-benylsubstituted, 3-substituted, 3,3disubstituted and oxa-cycloketone oximes were employed as substrates under the standard conditions. These oximes $\mathbf{2 b} \sim \mathbf{2 n}$ could go through the cyanoalkylsulfonylation/ cyclization smoothly. The corresponding cyanoalkylsulfonylated $9 H$-pyrrolo[1,2- $a$ ] indoles $\mathbf{3 a b} \sim \mathbf{3 a n}$ could be isolated in moderate yields. However, the substrates 20 and $\mathbf{2 p}$ were incompatible with the best conditions and most of substrate 1 was decomposed (product 3ao and 3ap).

To gain an insight of the mechanism for the tandem cyanoalkylsulfonylation/cyclization, we conducted a series of radical capture experiments (Scheme 3). The yields of $N$-propargylindoles $\mathbf{1 a}$ and cycloketone oxime esters $\mathbf{2 a}$ by utilizing 2,2,6,6-tetramethylpiperidinooxy (TEMPO), 2,6di- $t$-butyl- $p$-cresol (butylated hydroxytoluene) (BHT) or 1,1-diphenylethene as radical inhibitors under standard conditions were quite low (Scheme 3 ). The cyanoalkyl- 
Table 2 Scope of $N$-propargylindoles (1) and cycloketone oxime esters (2) ${ }^{a}$

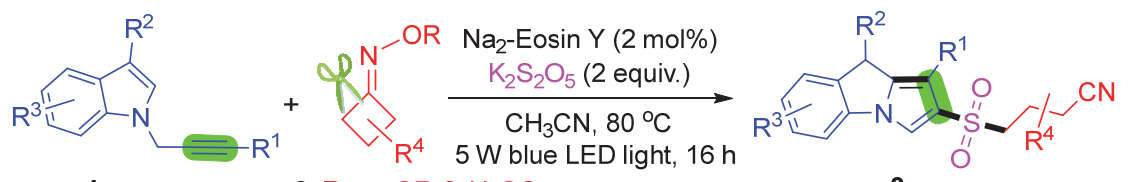

1

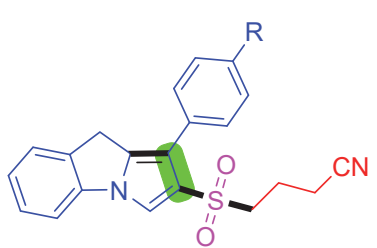

$\mathrm{R}=\mathrm{H}, \mathbf{3 a a}, 81 \%$

$\mathrm{R}=\mathrm{OMe}, \mathbf{3 b a}, 80 \%$

$\mathrm{R}=\mathrm{Me}, 3 \mathrm{ca}, 76 \%$

$\mathrm{R}={ }^{t} \mathrm{Bu}, 3 \mathrm{da}, 85 \%$

$\mathrm{R}=\mathrm{Ph}, 3$ ea, $82 \%$

$\mathrm{R}=\mathrm{F}, \mathbf{3 f a}, 80 \%$

$\mathrm{R}=\mathrm{Cl}, \mathbf{3 g a}, 78 \%$

$\mathrm{R}=\mathrm{CN}$ 3ha, $71 \%$

$\mathrm{R}=\mathrm{CF}_{3}, 3 \mathrm{ia}, 67 \%$

$\mathrm{R}=\mathrm{NO}_{2}, 3 \mathrm{ja}, 60 \%$

$\mathrm{R}$<smiles></smiles>

$\mathrm{R}=\mathrm{Me}, 3 \mathrm{ua}, 80 \%$

$\mathrm{R}=\mathrm{Cl}, 3 \mathbf{v a}, 76 \%$<smiles>N#CCCC(Cc1ccccc1)S(=O)(=O)c1cn2c(c1-c1ccccc1)Cc1ccccc1-2</smiles>

$3 a b, 66 \%$

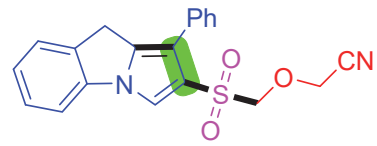

3an, $45 \%$

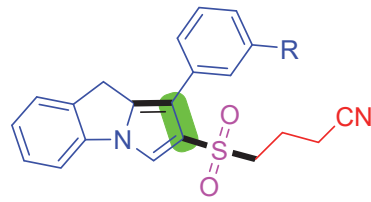

$\mathrm{R}=\mathrm{OMe}, \mathbf{3 k a}, 75 \%$

$\mathrm{R}=\mathrm{Me}, 3 \mathrm{la}, 72 \%$

$\mathrm{R}=\mathrm{NO}_{2}, 3 \mathrm{ma}, 54 \%$

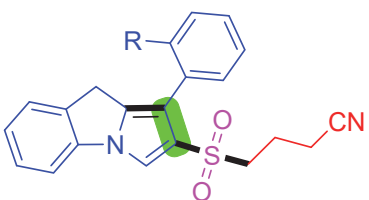

$\mathrm{R}=\mathrm{OMe}, 3 \mathrm{na}, 70 \%$

$\mathrm{R}=\mathrm{Me}, 30 \mathrm{a}, 65 \%$

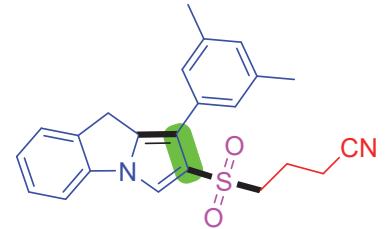

3pa, $69 \%$

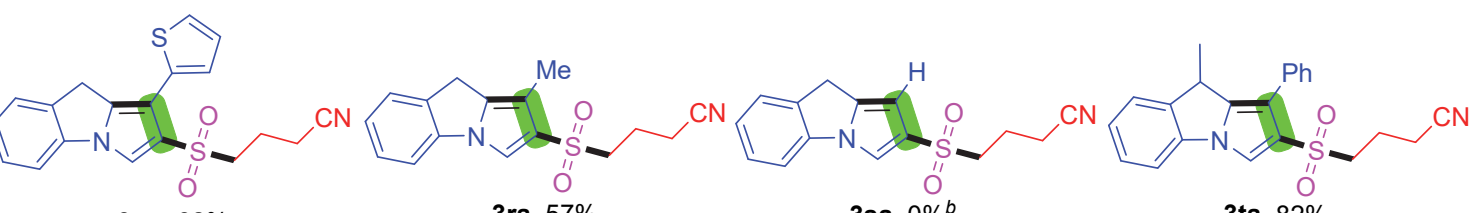

$3 q a, 62 \%$

3ra, $57 \%$

3sa, $0 \%^{b}$

3ta, $82 \%$<smiles>[R]c1ccc2c(c1)Cc1c(-c3ccccc3)c(S(=O)(=O)CCCC#N)cn1-2</smiles>

$\mathrm{R}=\mathrm{OMe}, 3 \mathbf{w a}, 82 \%$ $\mathrm{R}=\mathrm{Br}, 3 \mathbf{x a}, 72 \%$

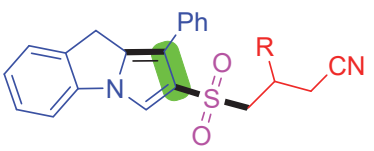

$\mathrm{R}=\mathrm{Ph}, \mathbf{3 a c}, 78 \%$

$\mathrm{R}=4-\mathrm{MeC}_{6} \mathrm{H}_{4}$, 3ad, $72 \%$

$\mathrm{R}=4{ }^{-}{ }^{\mathrm{B}} \mathrm{BuC}_{6} \mathrm{H}_{4}$, 3ae, 75\%

$\mathrm{R}=4-\mathrm{FC}_{6} \mathrm{H}_{4}$, 3af, $70 \%$

$\mathrm{R}=4-\mathrm{BrC}_{6} \mathrm{H}_{4}, 3 \mathrm{ag}, 66 \%$

$\mathrm{R}=\mathrm{Bn}, \mathbf{3 a h}, \mathbf{7 1} \%$

$\mathrm{R}=\mathrm{OBn}, \mathbf{3 a i}, 79 \%$

$\mathrm{R}=\mathrm{CO}_{2} \mathrm{Bn}, 3 \mathbf{a j}, 75 \%$

$\mathrm{R}=\mathrm{CO}_{2} \mathrm{Et}, 3 \mathrm{ak}, 72 \%$

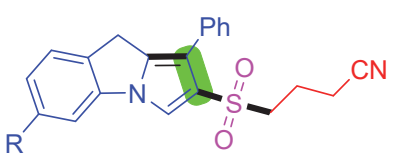

$\mathrm{R}=\mathrm{Me}, \mathbf{3 y a}, 83 \%$

$\mathrm{R}=\mathrm{F}, 3 \mathbf{z a}, 78 \%$

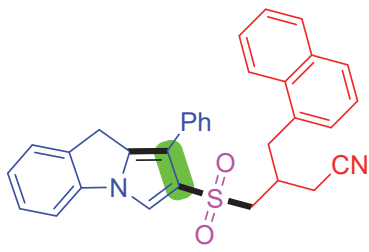

3al, $66 \%$

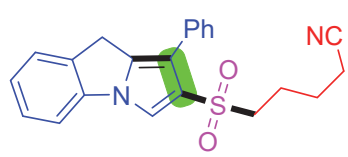

3ao, $0 \%^{b}$<smiles>Cc1cccc2c1-n1cc(S(=O)(=O)CCCC#N)c(-c3ccccc3)c1C2</smiles>

3aaa, $80 \%$<smiles>CC(CC#N)(CC(C)(c1ccccc1)S(=O)(=O)c1cn2c(c1-c1ccccc1)Cc1ccccc1-2)c1ccccc1</smiles>

3am, 60\%

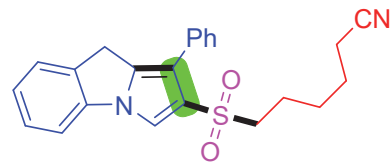

3ap, $0 \%^{b}$

${ }^{a}$ Reaction conditions: 1 ( $\left.0.2 \mathrm{mmol}\right), 2$ ( $0.3 \mathrm{mmol}, 1.5$ equiv.), $\mathrm{K}_{2} \mathrm{~S}_{2} \mathrm{O}_{5}\left(88.9 \mathrm{mg}, 0.4 \mathrm{mmol}, 2\right.$ equiv.), $\mathrm{Na}_{2}$-Eosin $\mathrm{Y}(2.8 \mathrm{mg}, 2 \mathrm{~mol} \%), \mathrm{CH}_{3} \mathrm{CN}(2 \mathrm{~mL})$ at $80{ }^{\circ} \mathrm{C}$ under an argon atmosphere and $5 \mathrm{~W}$ blue LED irradiation for $16 \mathrm{~h} .{ }^{b}$ Most of the substrate 1 was decomposed.

(a)<smiles>C(#Cc1ccccc1)Cn1ccc2ccccc21</smiles>

1a

$+\overbrace{}^{N-O R}$

2a

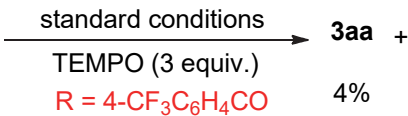

NC

$4,21 \%$, detected by GC-MS

(b)<smiles>C(#Cc1ccccc1)Cn1ccc2ccccc21</smiles>

1a
$\overbrace{\begin{array}{c}\mathrm{BHT}(3 \text { equiv. }) \\ \mathrm{R}=4-\mathrm{CF}_{3} \mathrm{C}_{6} \mathrm{H}_{4} \mathrm{CO}\end{array}}^{\stackrel{\text { standard conditions }}{N}}$

2a

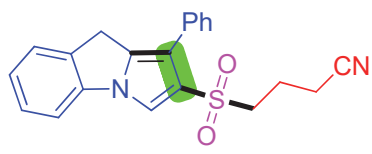

3aa, $5 \%$

(c)<smiles>C(#Cc1ccccc1)Cn1ccc2ccccc21</smiles>

1a<smiles>CCCCN=C1CCCC1</smiles>

2a $\frac{\text { standard conditions }}{1,1-\text { diphenylethene }(3 \text { equiv. })}$ 3aa + $\mathrm{R}=4-\mathrm{CF}_{3} \mathrm{C}_{6} \mathrm{H}_{4} \mathrm{CO}$

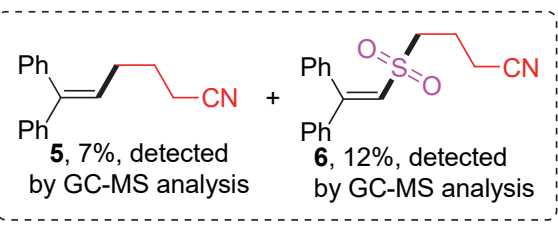

Scheme 3 Control experiments 
trapped product 4 could be detected by GC-MS analysis when the reaction was conducted by employing TEMPO as radical inhibitor (Scheme 3a). 1,1-Diphenylethene could trap the cyanoalkylsulfonyl radical and cyanoalkyl radical in the radical trapping reaction (Scheme 3c).

According to the above results and previous literature, ${ }^{[8,15-21]}$ a mechanism for the transition-metal-free visible-light-mediated tandem cyanoalkylsulfonylation/cyclization of alkynes with cycloketone oxime derivatives was proposed (Scheme 4). Initially, Eosin $\mathrm{Y}^{2^{-}}$was converted into Eosin $\mathrm{Y}^{2-*}$ photoexcited by the irradiation of light. ${ }^{[21 \mathrm{~d}, 21 \mathrm{f}]}$ Then, the iminyl radical $\mathbf{A}$, which generated from cycloketone oxime 2a under the reduction of Eosin $\mathrm{Y}^{2^{-}}$, underwent ring-opening to give cyanoalkyl radical B. Subsequently, the intermediate B reacted with $\mathrm{K}_{2} \mathrm{~S}_{2} \mathrm{O}_{5}$ to give cyanoalkylsulfonyl radical $\mathbf{C} .^{[8 \mathrm{a}-8 \mathrm{~d}]}$ The intermediate $\mathbf{C}$ attacked the carbon-carbon triple bond in $\mathrm{N}$-propargylindoles 1a to furnish the radical $\mathbf{D}$, which underwent intramolecular cyclization to give the radical E. Next, the radical $\mathbf{E}$ went through SET by Eosin $\mathrm{Y}^{-}$to provide the carbocation $\mathbf{F}$, and Eosin $\mathrm{Y}^{-}$was reverted to Eosin $\mathrm{Y}^{2-}$. Finally, the intermediate $\mathbf{F}$ underwent deprotonation and isomerization to form the target product 3aa. ${ }^{[17]}$

\section{Conclusions}

In conclusion, we have established a transition-metalfree visible-light-mediated tandem cyanoalkylsulfonylation/cyclization of alkynes with cycloketone oxime derivatives for the construction of 2-cyanoalkylsulfonyl-9Hpyrrolo[1,2- $a$ ] indoles through the insertion of $\mathrm{SO}_{2}$. This method is the first example by using alkynes as radical acceptors for trapping cyanoalkylsulfonyl radicals under transition-metal-free conditions. This difuctionalization of alkynes involves a radical pathway, which undergoes for- mation of iminyl radical, ring-opening of four-membered carbocycle, insertion of $\mathrm{SO}_{2}$, addition of sulfonyl radical to alkyne, cyclization and isomerization. This strategy features a broad substrate scope, simple reaction conditions and common $\mathrm{SO}_{2}$ source. The further study and application of the cyanoalkylsulfonylation/cyclization reaction are currently undergoing in our laboratory.

\section{Experimental section}

The ${ }^{1} \mathrm{H}$ NMR and ${ }^{13} \mathrm{C}$ NMR spectra were recorded in $\mathrm{CDCl}_{3}$ solvent on an NMR spectrometer using TMS as internal standard. LRMS was performed on a GC-MS instrument and HRMS was measured on an electrospray ionization (ESI) apparatus using time-of-flight (TOF) mass spectrometry. Melting points are uncorrected.

\subsection{Preparation of $\mathrm{N}$-propargylindoles 1 and cyclo- butanone oxime esters 2}

All of the $N$-propargylindoles $\mathbf{1}^{[21]}$ and cyclobutanone oxime esters $2^{[5-8]}$ were synthesized according to the known methods.

\subsection{Typical experimental procedure for the cyano- alkylsulfonylation/cyclization reaction}

To a Schlenk tube were added $N$-propargylindoles $1(0.2$ mmol), cyclobutanone oxime esters 2 (1.5 equiv., 0.3 mmol), $\mathrm{Na}_{2}$-Eosin $\mathrm{Y}$ (2.8 $\mathrm{mg}, 0.004 \mathrm{mmol}, 2 \mathrm{~mol} \%$ ), $\mathrm{K}_{2} \mathrm{~S}_{2} \mathrm{O}_{5}$ (88.9 mg, $0.4 \mathrm{mmol}, 2$ equiv.) and $\mathrm{CH}_{3} \mathrm{CN}(2 \mathrm{~mL})$. Then the tube was stirred at $80{ }^{\circ} \mathrm{C}$ in Ar atmosphere under $5 \mathrm{~W}$ blue LED light for the indicated time until complete consumption of starting material as monitored by thinlayer chromatography (TLC) analysis. After the reaction was finished, the reaction mixture was washed with brine. The aqueous phase was re-extracted with EtOAc $(10 \mathrm{~mL} \times$

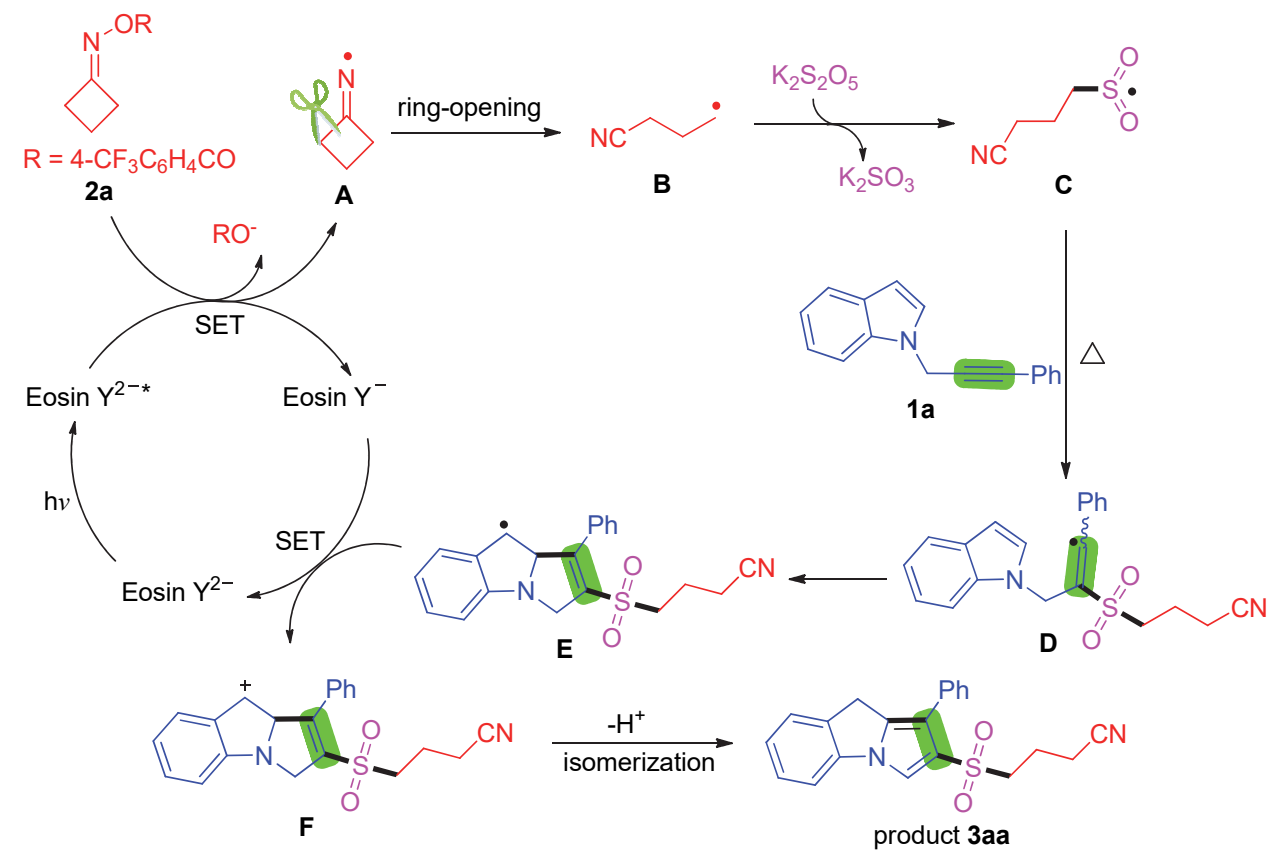

Scheme 4 Possible mechanisms 
3 ). The combined organic extracts were dried over $\mathrm{Na}_{2} \mathrm{SO}_{4}$ and concentrated in vacuum. The residue was purified by silica gel flash column chromatography [hexane/ethyl acetate $(V: V=5: 1$ to $3: 1)]$ to afford the desired products 3.

4-((1-Phenyl-9H-pyrrolo[1,2-a]indol-2-yl)sulfonyl)butanenitrile (3aa): $58.3 \mathrm{mg}, 81 \%$ yield. Yellow solid, m.p. $103.3 \sim 103.8{ }^{\circ} \mathrm{C} ;{ }^{1} \mathrm{H}$ NMR $\left(400 \mathrm{MHz}, \mathrm{CDCl}_{3}\right) \delta: 7.86(\mathrm{~s}$, $1 \mathrm{H}), 7.68 \sim 7.66(\mathrm{~m}, 2 \mathrm{H}), 7.48 \sim 7.35(\mathrm{~m}, 6 \mathrm{H}), 7.28 \sim 7.24$ $(\mathrm{m}, 1 \mathrm{H}), 3.98(\mathrm{~s}, 2 \mathrm{H}), 2.97(\mathrm{t}, J=7.2 \mathrm{~Hz}, 2 \mathrm{H}), 2.41(\mathrm{t}, J=$ $7.2 \mathrm{~Hz}, 2 \mathrm{H}), 1.97$ (t, $J=7.2 \mathrm{~Hz}, 2 \mathrm{H}) ;{ }^{13} \mathrm{C} \mathrm{NMR}(100 \mathrm{MHz}$, $\left.\mathrm{CDCl}_{3}\right) \delta: 139.2,135.5,133.9,132.0,129.3,128.7,128.1$, $127.7,126.2,125.5,123.6,118.3,116.9,116.5,111.0$, 53.5, 29.3, 19.4, 15.9; HRMS (ESI-TOF) calcd for $\mathrm{C}_{21} \mathrm{H}_{19^{-}}$ $\mathrm{N}_{2} \mathrm{O}_{2} \mathrm{~S}(\mathrm{M}+\mathrm{H})^{+}$363.1162, found 363.1167.

4-((1-(4-Methoxyphenyl)-9H-pyrrolo[1,2-a]indol-2-yl)sulfonyl)butanenitrile (3ba): $62.7 \mathrm{mg}, 80 \%$ yield, gray oil. ${ }^{1} \mathrm{H}$ NMR (400 MHz, $\left.\mathrm{CDCl}_{3}\right) \delta: 7.84(\mathrm{~s}, 1 \mathrm{H}), 7.61 \sim 7.58$ $(\mathrm{m}, 2 \mathrm{H}), 7.47(\mathrm{~d}, J=7.2 \mathrm{~Hz}, 1 \mathrm{H}), 7.42 \sim 7.41(\mathrm{~m}, 2 \mathrm{H})$, $7.28 \sim 7.26(\mathrm{~m}, 1 \mathrm{H}), 7.01 \sim 6.98(\mathrm{~m}, 2 \mathrm{H}), 3.96(\mathrm{~s}, 2 \mathrm{H})$, $3.86(\mathrm{~s}, 3 \mathrm{H}), 2.97(\mathrm{t}, J=7.2 \mathrm{~Hz}, 2 \mathrm{H}), 2.42$ (t, $J=7.2 \mathrm{~Hz}$, 2H), $2.01 \sim 1.94(\mathrm{~m}, 2 \mathrm{H}) ;{ }^{13} \mathrm{C}$ NMR $\left(100 \mathrm{MHz}, \mathrm{CDCl}_{3}\right) \delta$ : $159.1,139.3,135.2,134.0,130.6,128.1,126.3,125.5$, $124.2,123.6,118.3,116.7,116.2,114.1,111.0,55.3,53.5$, 29.3, 19.4, 16.0; HRMS (ESI-TOF) calcd for $\mathrm{C}_{22} \mathrm{H}_{21} \mathrm{~N}_{2} \mathrm{O}_{3} \mathrm{~S}$ $(\mathrm{M}+\mathrm{H})^{+}$393.1267, found 393.1272.

4-((1-( $p$-Tolyl)-9H-pyrrolo[1,2-a]indol-2-yl)sulfonyl)butanenitrile (3ca): $57.2 \mathrm{mg}, 76 \%$ yield. Yellow solid, m.p. $110.3 \sim 110.8{ }^{\circ} \mathrm{C}$; ${ }^{1} \mathrm{H}$ NMR $\left(400 \mathrm{MHz}, \mathrm{CDCl}_{3}\right) \delta: 7.85$ (s, $1 \mathrm{H}), 7.55(\mathrm{~d}, J=8.0 \mathrm{~Hz}, 2 \mathrm{H}), 7.47(\mathrm{~d}, J=7.6 \mathrm{~Hz}, 1 \mathrm{H})$, $7.44 \sim 7.39(\mathrm{~m}, 2 \mathrm{H}), 7.29 \sim 7.24(\mathrm{~m}, 3 \mathrm{H}), 3.97(\mathrm{~s}, 2 \mathrm{H})$, $2.97(\mathrm{t}, J=7.2 \mathrm{~Hz}, 2 \mathrm{H}), 2.41(\mathrm{t}, J=3.2 \mathrm{~Hz}, 5 \mathrm{H}), 2.01 \sim$ $1.94(\mathrm{~m}, 2 \mathrm{H}) ;{ }^{13} \mathrm{C}$ NMR $\left(100 \mathrm{MHz}, \mathrm{CDCl}_{3}\right) \delta$ : 139.3, $137.6,135.3,134.0,129.4,129.2,129.0,128.1,126.3$, $125.5,123.6,118.3,117.0,116.3,111.0,53.5,29.3,21.3$, 19.4, 16.0; HRMS (ESI-TOF) calcd for $\mathrm{C}_{22} \mathrm{H}_{21} \mathrm{~N}_{2} \mathrm{O}_{2} \mathrm{~S}$ $(\mathrm{M}+\mathrm{H})^{+} 377.1318$, found 377.1323 .

4-((1-(4-(tert-Butyl)phenyl)-9H-pyrrolo[1,2-a]indol-2yl)sulfonyl)butanenitrile (3da): $66.9 \mathrm{mg}, 85 \%$ yield, gray oil. ${ }^{1} \mathrm{H}$ NMR (400 MHz, $\left.\mathrm{CDCl}_{3}\right) \delta: 7.86(\mathrm{~s}, 1 \mathrm{H}), 7.60(\mathrm{~d}$, $J=8.4 \mathrm{~Hz}, 2 \mathrm{H}), 7.47(\mathrm{~d}, J=8.4 \mathrm{~Hz}, 3 \mathrm{H}), 7.44 \sim 7.39(\mathrm{~m}$, 2H), $7.28 \sim 7.24(\mathrm{~m}, 1 \mathrm{H}), 4.00(\mathrm{~s}, 2 \mathrm{H}), 2.98(\mathrm{t}, J=6.8 \mathrm{~Hz}$, $2 \mathrm{H}), 2.41(\mathrm{t}, J=7.2 \mathrm{~Hz}, 2 \mathrm{H}), 2.01 \sim 1.96(\mathrm{~m}, 2 \mathrm{H}), 1.37(\mathrm{~s}$, $9 \mathrm{H}) ;{ }^{13} \mathrm{C}$ NMR $\left(100 \mathrm{MHz}, \mathrm{CDCl}_{3}\right) \delta: 150.6,139.3,135.4$, $134.0,129.0,128.9,128.1,126.2,125.6,125.5,123.6$, $118.3,116.9,116.4,111.0,53.5,34.6,31.3,29.5,19.4$, 15.9; HRMS (ESI-TOF) calcd for $\mathrm{C}_{25} \mathrm{H}_{27} \mathrm{~N}_{2} \mathrm{O}_{2} \mathrm{~S}(\mathrm{M}+\mathrm{H})^{+}$ 419.1788, found 419.1794.

4-((1-([1,1'-Biphenyl]-4-yl)-9H-pyrrolo[1,2- $a]$ indol-2yl)sulfonyl)butanenitrile (3ea): $71.8 \mathrm{mg}, \quad 82 \%$ yield. Yellow solid, m.p. 112.0 112.6 ${ }^{\circ} \mathrm{C} ;{ }^{1} \mathrm{H}$ NMR (400 MHz, $\left.\mathrm{CDCl}_{3}\right) \delta: 7.89(\mathrm{~s}, 1 \mathrm{H}), 7.77 \sim 7.75(\mathrm{~m}, 2 \mathrm{H}), 7.70 \sim 7.65$ $(\mathrm{m}, 4 \mathrm{H}), 7.50 \sim 7.42(\mathrm{~m}, 5 \mathrm{H}), 7.40 \sim 7.35(\mathrm{~m}, 1 \mathrm{H}), 7.29 \sim$ $7.27(\mathrm{~m}, 1 \mathrm{H}), 4.03(\mathrm{~s}, 2 \mathrm{H}), 3.03(\mathrm{t}, J=7.2 \mathrm{~Hz}, 2 \mathrm{H}), 2.44$ (t, $J=7.2 \mathrm{~Hz}, 2 \mathrm{H}), 2.04 \sim 1.97(\mathrm{~m}, 2 \mathrm{H}) ;{ }^{13} \mathrm{C} \mathrm{NMR}(100 \mathrm{MHz}$, $\left.\mathrm{CDCl}_{3}\right) \delta: 140.4,140.3,139.2,135.7,133.9,131.0,129.7$, $128.8,128.1,127.5,127.3,127.0,126.3,125.6,123.6$,
118.3, 116.7, 116.6, 111.0, 53.6, 29.5, 19.4, 16.0; HRMS (ESI-TOF) calcd for $\mathrm{C}_{27} \mathrm{H}_{23} \mathrm{~N}_{2} \mathrm{O}_{2} \mathrm{~S}(\mathrm{M}+\mathrm{H})^{+}$439.1475, found 439.1480 .

4-((1-(4-Fluorophenyl)-9H-pyrrolo[1,2-a]indol-2-yl)sulfonyl)butanenitrile (3fa): $64.6 \mathrm{mg}, 85 \%$ yield. Yellow solid, m.p. 127.3 127.8 ${ }^{\circ} \mathrm{C}$; ${ }^{1} \mathrm{H}$ NMR (400 $\mathrm{MHz} \mathrm{CDCl}_{3}$ ) $\delta: 7.86(\mathrm{~s}, 1 \mathrm{H}), 7.66 \sim 7.62(\mathrm{~m}, 2 \mathrm{H}), 7.48(\mathrm{~d}, J=7.2 \mathrm{~Hz}$, $1 \mathrm{H}), 7.45 \sim 7.40(\mathrm{~m}, 2 \mathrm{H}), 7.31 \sim 7.24(\mathrm{~m}, 1 \mathrm{H}), 7.18 \sim 7.12$ $(\mathrm{m}, 2 \mathrm{H}), 3.96(\mathrm{~s}, 2 \mathrm{H}), 2.97(\mathrm{t}, J=7.2 \mathrm{~Hz}, 2 \mathrm{H}), 2.44(\mathrm{t}, J=$ $7.2 \mathrm{~Hz}, 2 \mathrm{H}), 2.03 \sim 1.96(\mathrm{~m}, 2 \mathrm{H}) ;{ }^{13} \mathrm{C} \mathrm{NMR}(100 \mathrm{MHz}$, $\left.\mathrm{CDCl}_{3}\right) \delta: 162.3(\mathrm{~d}, J=246.2 \mathrm{~Hz}, 1 \mathrm{C}), 139.2,135.6,133.8$, $131.2(\mathrm{~d}, J=8.0 \mathrm{~Hz}, 1 \mathrm{C}), 128.2,128.0(\mathrm{~d}, \quad J=3.4 \mathrm{~Hz}$, 1C), 126.3, 125.6, 123.6, 118.2, 116.5, 116.1, 115.7 (d, $J=$ $21.3 \mathrm{~Hz}, 1 \mathrm{C}), 111.0,53.7,29.2,19.4,16.0 ;{ }^{19} \mathrm{~F}$ NMR (282 $\left.\mathrm{MHz} \mathrm{CDCl}_{3}\right) \delta$ : -113.8 (s, 1F); HRMS (ESI-TOF) calcd for $\mathrm{C}_{21} \mathrm{H}_{18}{ }^{19} \mathrm{FN}_{2} \mathrm{O}_{2} \mathrm{~S}(\mathrm{M}+\mathrm{H})^{+}$381.1068, found 381.1074.

4-((1-(4-Chlorophenyl)-9H-pyrrolo[1,2-a]indol-2-yl)sulfonyl)butanenitrile (3ga): $59.3 \mathrm{mg}, 78 \%$ yield. Yellow solid, m.p. $182.1 \sim 182.6{ }^{\circ} \mathrm{C} ;{ }^{1} \mathrm{H}$ NMR (400 MHz, $\mathrm{CDCl}_{3}$ ) $\delta: 7.87(\mathrm{~s}, 1 \mathrm{H}), 7.63 \sim 7.61(\mathrm{~m}, 2 \mathrm{H}), 7.48(\mathrm{~d}, J=7.6 \mathrm{~Hz}$, $1 \mathrm{H}), 7.44 \sim 7.42(\mathrm{~m}, 4 \mathrm{H}), 7.30 \sim 7.26(\mathrm{~m}, 1 \mathrm{H}), 3.97(\mathrm{~s}$, $2 \mathrm{H}), 2.99$ (t, $J=7.2 \mathrm{~Hz}, 2 \mathrm{H}), 2.45$ (t, $J=7.2 \mathrm{~Hz}, 2 \mathrm{H})$, $2.04 \sim 1.96(\mathrm{~m}, 2 \mathrm{H}) ;{ }^{13} \mathrm{C} \mathrm{NMR}\left(100 \mathrm{MHz}, \mathrm{CDCl}_{3}\right) \delta$ : $139.1,135.8,133.8,133.7,130.7,130.5,128.9,128.2$, $126.3,125.7,123.6,118.2,116.8,115.9,111.1,53.8,29.4$, 19.4, 16.0; HRMS (ESI-TOF) calcd for $\mathrm{C}_{21} \mathrm{H}_{18}{ }^{35} \mathrm{ClN}_{2} \mathrm{O}_{2} \mathrm{~S}$ $(\mathrm{M}+\mathrm{H})^{+}$396.0699, found 396.0705.

4-(2-((3-Cyanopropyl)sulfonyl)-9H-pyrrolo[1,2-a]indol1-yl)benzonitrile (3ha): $55.0 \mathrm{mg}, 71 \%$ yield. Yellow solid, m.p. $210.2 \sim 210.8{ }^{\circ} \mathrm{C} ;{ }^{1} \mathrm{H}$ NMR (400 $\left.\mathrm{MHz} \mathrm{CDCl}_{3}\right) \delta$ : $7.91(\mathrm{~s}, 1 \mathrm{H}), 7.84(\mathrm{~d}, J=8.0 \mathrm{~Hz}, 2 \mathrm{H}), 7.75(\mathrm{~d}, \quad J=8.0 \mathrm{~Hz}$, $2 \mathrm{H}), 7.51(\mathrm{~d}, J=7.6 \mathrm{~Hz}, 1 \mathrm{H}), 7.48 \sim 7.45(\mathrm{~m}, 2 \mathrm{H}), 7.33 \sim$ $7.29(\mathrm{~m}, 1 \mathrm{H}), 4.02(\mathrm{~s}, 2 \mathrm{H}), 3.01(\mathrm{t}, J=7.2 \mathrm{~Hz}, 2 \mathrm{H}), 2.47(\mathrm{t}$, $J=7.2 \mathrm{~Hz}, 2 \mathrm{H}), 2.06 \sim 1.99(\mathrm{~m}, 2 \mathrm{H}) ;{ }^{13} \mathrm{C} \mathrm{NMR}(100 \mathrm{MHz}$, $\left.\mathrm{CDCl}_{3}\right) \delta: 139.0,137.1,136.6,133.5,132.5,129.9,128.4$, $126.3,126.0,123.7,118.7,118.1,117.6,115.4,111.2$, 111.2, 54.1, 29.7, 19.3, 16.0; HRMS (ESI-TOF) calcd for $\mathrm{C}_{22} \mathrm{H}_{18} \mathrm{~N}_{3} \mathrm{O}_{2} \mathrm{~S}(\mathrm{M}+\mathrm{H})^{+}$388.1114, found 388.1119.

4-((1-(4-(Trifluoromethyl)phenyl)-9H-pyrrolo[1,2a]indol-2-yl)sulfonyl)butanenitrile (3ia): $57.6 \mathrm{mg}, 67 \%$ yield. Yellow solid, m.p. $188.2 \sim 188.7{ }^{\circ} \mathrm{C} ;{ }^{1} \mathrm{H}$ NMR $(400$ $\left.\mathrm{MHz}, \mathrm{CDCl}_{3}\right) \delta: 7.90(\mathrm{~s}, 1 \mathrm{H}), 7.82(\mathrm{~d}, J=8.0 \mathrm{~Hz}, 2 \mathrm{H})$, $7.71(\mathrm{~d}, J=8.0 \mathrm{~Hz}, 2 \mathrm{H}), 7.50(\mathrm{~d}, J=7.6 \mathrm{~Hz}, 1 \mathrm{H}), 7.47 \sim$ $7.42(\mathrm{~m}, 2 \mathrm{H}), 7.31 \sim 7.26(\mathrm{~m}, 1 \mathrm{H}), 4.00(\mathrm{~s}, 2 \mathrm{H}), 3.00(\mathrm{t}$, $J=7.2 \mathrm{~Hz}, 2 \mathrm{H}), 2.46(\mathrm{t}, J=7.2 \mathrm{~Hz}, 2 \mathrm{H}), 2.05 \sim 1.98(\mathrm{~m}$, $2 \mathrm{H}) ;{ }^{13} \mathrm{C} \mathrm{NMR}\left(100 \mathrm{MHz}, \mathrm{CDCl}_{3}\right) \delta: 139.1,136.3,135.9$ (d, $J=1.4 \mathrm{~Hz}, 1 \mathrm{C}), 133.7,129.7$ (d, $J=32.2 \mathrm{~Hz}, 1 \mathrm{C})$, $129.6,128.2(\mathrm{~d}, J=15.3,1 \mathrm{C}), 126.3,125.8,125.6(\mathrm{q}, J=$ $3.7 \mathrm{~Hz}, 1 \mathrm{C}$ ), 124.1 (q, $J=270.3 \mathrm{~Hz}, 1 \mathrm{C}), 123.6,118.2$, $117.2,115.7,111.1,54.0,29.5,19.4,16.0 ;{ }^{19} \mathrm{~F}$ NMR $(282$ $\left.\mathrm{MHz}, \mathrm{CDCl}_{3}\right) \delta$ : -62.5 (s, 3F); HRMS (ESI-TOF) calcd for $\mathrm{C}_{22} \mathrm{H}_{18}{ }^{19} \mathrm{~F}_{3} \mathrm{~N}_{2} \mathrm{O}_{2} \mathrm{~S}(\mathrm{M}+\mathrm{H})^{+}$431.1036, found 431.1042.

4-((1-(4-Nitrophenyl)-9H-pyrrolo[1,2-a]indol-2-yl)sulfonyl)butanenitrile (3ja): $48.8 \mathrm{mg}, 60 \%$ yield. Yellow solid, m.p. 210.3 210.6 ${ }^{\circ} \mathrm{C} ;{ }^{1} \mathrm{H}$ NMR (400 MHz, $\mathrm{CDCl}_{3}$ ) $\delta: 8.33 \sim 8.29(\mathrm{~m}, 2 \mathrm{H}), 7.93 \sim 7.90(\mathrm{~m}, 3 \mathrm{H}), 7.53 \sim 7.46$ $(\mathrm{m}, 3 \mathrm{H}), 7.34 \sim 7.30(\mathrm{~m}, 1 \mathrm{H}), 4.04(\mathrm{~s}, 2 \mathrm{H}), 3.03(\mathrm{t}, J=7.2$ 
$\mathrm{Hz}, 2 \mathrm{H}), 2.48(\mathrm{t}, J=7.2 \mathrm{~Hz}, 2 \mathrm{H}), 2.07 \sim 2.00(\mathrm{~m}, 2 \mathrm{H}) ;{ }^{13} \mathrm{C}$ NMR $\left(100 \mathrm{MHz}, \mathrm{CDCl}_{3}\right) \delta: 146.9,139.1,138.9,136.9$, $133.5,130.0,128.4,126.3,126.1,124.0,123.8,118.1$, $117.8,115.1,111.2,54.2,29.7,19.3,16.0$; HRMS (ESITOF) calcd for $\mathrm{C}_{21} \mathrm{H}_{18} \mathrm{~N}_{3} \mathrm{O}_{4} \mathrm{~S}(\mathrm{M}+\mathrm{H})^{+} 408.1013$, found 408.1018.

4-((1-(3-Methoxyphenyl)-9H-pyrrolo[1,2- $a$ ]indol-2-yl)sulfonyl)butanenitrile (3ka): $58.8 \mathrm{mg}, 75 \%$ yield. Yellow solid, m.p. 158.3 158.9 ${ }^{\circ} \mathrm{C}$; ${ }^{1} \mathrm{H}$ NMR (400 MHz, $\mathrm{CDCl}_{3}$ ) $\delta: 7.87(\mathrm{~s}, 1 \mathrm{H}), 7.48(\mathrm{~d}, J=7.2 \mathrm{~Hz}, 1 \mathrm{H}), 7.43 \sim 7.42(\mathrm{~m}$, 2H), $7.37(\mathrm{t}, J=8.0 \mathrm{~Hz}, 1 \mathrm{H}), 7.31 \sim 7.30(\mathrm{~m}, 1 \mathrm{H}), 7.26 \sim$ $7.22(\mathrm{~m}, 2 \mathrm{H}), 6.93 \sim 6.91(\mathrm{~m}, 1 \mathrm{H}), 4.00(\mathrm{~s}, 2 \mathrm{H}), 3.86(\mathrm{~s}$, $3 \mathrm{H}), 3.00(\mathrm{t}, J=7.2 \mathrm{~Hz}, 2 \mathrm{H}), 2.43(\mathrm{t}, J=7.2 \mathrm{~Hz}, 2 \mathrm{H})$, $2.02 \sim 1.95(\mathrm{~m}, 2 \mathrm{H}) ;{ }^{13} \mathrm{C} \mathrm{NMR}\left(100 \mathrm{MHz}, \mathrm{CDCl}_{3}\right) \delta$ : $159.7,139.2,135.6,133.9,133.4,129.7,128.1,126.3$, $125.6,123.6,121.4,118.3,116.8,116.6,114.7,113.6$, 1110, 55.3, 53.5, 29.5, 19.4, 16.0; HRMS (ESI-TOF) calcd for $\mathrm{C}_{22} \mathrm{H}_{21} \mathrm{~N}_{2} \mathrm{O}_{3} \mathrm{~S}(\mathrm{M}+\mathrm{H})^{+}$393.1267, found 393.1273.

4-((1-(m-Tolyl)-9H-pyrrolo[1,2- $a$ ]indol-2-yl)sulfonyl)butanenitrile (3la): $54.1 \mathrm{mg}, 72 \%$ yield. Yellow solid, m.p. $139.0 \sim 139.6{ }^{\circ} \mathrm{C} ;{ }^{1} \mathrm{H}$ NMR $\left(400 \mathrm{MHz}, \mathrm{CDCl}_{3}\right) \delta: 7.86(\mathrm{~s}$, $1 \mathrm{H}), 7.48 \sim 7.46(\mathrm{~m}, 3 \mathrm{H}), 7.44 \sim 7.40(\mathrm{~m}, 2 \mathrm{H}), 7.34(\mathrm{t}, J=$ $8.0 \mathrm{~Hz}, 1 \mathrm{H}), 7.28 \sim 7.24(\mathrm{~m}, 1 \mathrm{H}), 7.19(\mathrm{~d}, J=7.2 \mathrm{~Hz}, 1 \mathrm{H})$, $3.98(\mathrm{~s}, 2 \mathrm{H}), 2.97(\mathrm{t}, J=7.2 \mathrm{~Hz}, 2 \mathrm{H}), 2.41(\mathrm{t}, J=7.2 \mathrm{~Hz}$, 5H), $2.01 \sim 1.94(\mathrm{~m}, 2 \mathrm{H}) ;{ }^{13} \mathrm{C} \mathrm{NMR}\left(100 \mathrm{MHz}, \mathrm{CDCl}_{3}\right) \delta$ : $139.3,138.3,135.5,134.0,132.0,129.9,128.6,128.5$, $128.1,126.4,126.2,125.5,123.7,118.3,117.1,116.4$, 111.0, 53.5, 29.4, 21.5, 19.4, 15.9; HRMS (ESI-TOF) calcd for $\mathrm{C}_{22} \mathrm{H}_{21} \mathrm{~N}_{2} \mathrm{O}_{2} \mathrm{~S}(\mathrm{M}+\mathrm{H}){ }^{+}$377.1318, found 377.1323 .

4-((1-(3-Nitrophenyl)-9H-pyrrolo[1,2-a]indol-2-yl)sulfonyl)butanenitrile (3ma): $44.0 \mathrm{mg}, 54 \%$ yield. Yellow solid, m.p. $119.8 \sim 120.3{ }^{\circ} \mathrm{C}$; ${ }^{1} \mathrm{H}$ NMR (400 MHz, $\mathrm{CDCl}_{3}$ ) $\delta: 8.50(\mathrm{t}, J=2.0 \mathrm{~Hz}, 1 \mathrm{H}), 8.23 \sim 8.21(\mathrm{~m}, 1 \mathrm{H}), 8.14(\mathrm{~d}$, $J=8.0 \mathrm{~Hz}, 1 \mathrm{H}), 7.91(\mathrm{~s}, 1 \mathrm{H}), 7.65(\mathrm{t}, J=8.0 \mathrm{~Hz}, 1 \mathrm{H}), 7.50$ $(\mathrm{d}, J=7.6 \mathrm{~Hz}, 1 \mathrm{H}), 7.47 \sim 7.45(\mathrm{~m}, 2 \mathrm{H}), 7.33 \sim 7.30(\mathrm{~m}$, 1H), 4.04 (s, 2H), 3.03 (t, J=7.2 Hz, 2H), 2.49 (t, $J=7.2$ $\mathrm{Hz}, 2 \mathrm{H}), 2.08 \sim 2.01(\mathrm{~m}, 2 \mathrm{H}) ;{ }^{13} \mathrm{C} \mathrm{NMR}(100 \mathrm{MHz}$, $\left.\mathrm{CDCl}_{3}\right) \delta: 148.4,139.0,136.6,135.7,133.9,133.5,129.7$, $128.3,126.4,126.0,123.7,123.6,122.5,118.1,117.4$, $114.8,111.2,54.3,29.5,19.3,16.0$; HRMS (ESI-TOF) calcd for $\mathrm{C}_{21} \mathrm{H}_{18} \mathrm{~N}_{3} \mathrm{O}_{4} \mathrm{~S}(\mathrm{M}+\mathrm{H})^{+}$408.1013, found 408.1018.

4-((1-(2-Methoxyphenyl)-9H-pyrrolo[1,2-a]indol-2-yl)sulfonyl)butanenitrile (3na): $54.9 \mathrm{mg}, 70 \%$ yield. Yellow solid, m.p. 112.0 112.6 ${ }^{\circ} \mathrm{C}$; ${ }^{1} \mathrm{H}$ NMR (400 MHz, $\left.\mathrm{CDCl}_{3}\right)$ $\delta: 7.84(\mathrm{~s}, 1 \mathrm{H}), 7.59 \sim 7.56(\mathrm{~m}, 1 \mathrm{H}), 7.47 \sim 7.38(\mathrm{~m}, 4 \mathrm{H})$, $7.27 \sim 7.24(\mathrm{~m}, 1 \mathrm{H}), 7.07 \sim 7.05(\mathrm{~m}, 1 \mathrm{H}), 7.04 \sim 7.00(\mathrm{~m}$, $1 \mathrm{H}), 3.85(\mathrm{~s}, 3 \mathrm{H}), 3.79(\mathrm{~s}, 2 \mathrm{H}), 2.95(\mathrm{t}, J=7.2 \mathrm{~Hz}, 2 \mathrm{H})$, $2.36(\mathrm{t}, J=7.6 \mathrm{~Hz}, 2 \mathrm{H}), 2.01 \sim 1.93(\mathrm{~m}, 2 \mathrm{H}) ;{ }^{13} \mathrm{C} \mathrm{NMR}$ $\left(100 \mathrm{MHz}, \mathrm{CDCl}_{3}\right) \delta: 156.9,139.5,136.7,134.6,133.0$, $129.6,127.9,126.2,125.3,124.0,120.5,120.0,118.4$, $115.7,112.6,111.0,110.6,55.4,53.8,29.6,19.5,15.9$; HRMS (ESI-TOF) calcd for $\mathrm{C}_{22} \mathrm{H}_{21} \mathrm{~N}_{2} \mathrm{O}_{3} \mathrm{~S}(\mathrm{M}+\mathrm{H})^{+}$ 393.1267, found 393.1274.

4-((1-(o-Tolyl)-9H-pyrrolo[1,2- $a$ ]indol-2-yl)sulfonyl)butanenitrile (30a): $48.9 \mathrm{mg}, 65 \%$ yield, yellow oil. ${ }^{1} \mathrm{H}$
NMR (400 MHz, $\left.\mathrm{CDCl}_{3}\right) \delta: 7.85(\mathrm{~s}, 1 \mathrm{H}), 7.46 \sim 7.42(\mathrm{~m}$, $3 \mathrm{H}), 7.33 \sim 7.31(\mathrm{~m}, 3 \mathrm{H}), 7.28 \sim 7.23(\mathrm{~m}, 2 \mathrm{H}), 3.80 \sim 3.67$ $(\mathrm{m}, 2 \mathrm{H}), 2.93 \sim 2.89(\mathrm{~m}, 2 \mathrm{H}), 2.45 \sim 2.42(\mathrm{~m}, 2 \mathrm{H}), 2.42(\mathrm{~s}$, $3 \mathrm{H}), 2.03 \sim 1.96(\mathrm{~m}, 2 \mathrm{H}) ;{ }^{13} \mathrm{C}$ NMR $\left(100 \mathrm{MHz}, \mathrm{CDCl}_{3}\right) \delta$ : $139.4,138.0,135.5,134.2,131.0,130.9,130.1,128.5$, $128.1,126.3,125.5,125.4,124.1,118.3,116.2,115.5$, $111.0,54.0,28.9,20.1,19.4,16.0$; HRMS (ESI-TOF) calcd for $\mathrm{C}_{22} \mathrm{H}_{21} \mathrm{~N}_{2} \mathrm{O}_{2} \mathrm{~S}(\mathrm{M}+\mathrm{H})^{+}$377.1318, found 377.1323 .

4-((1-(3,5-Dimethylphenyl)-9H-pyrrolo[1,2- $a$ ]indol-2yl)sulfonyl)butanenitrile (3pa): $53.8 \mathrm{mg}, 80 \%$ yield. Yellow solid, m.p. 147.6 148.1 ${ }^{\circ} \mathrm{C}$; ${ }^{1} \mathrm{H}$ NMR (400 MHz, $\left.\mathrm{CDCl}_{3}\right) \delta: 7.85(\mathrm{~s}, 1 \mathrm{H}), 7.47(\mathrm{~d}, J=7.6 \mathrm{~Hz}, 1 \mathrm{H}), 7.43 \sim$ $7.39(\mathrm{~m}, 2 \mathrm{H}), 7.28 \sim 7.24(\mathrm{~m}, 3 \mathrm{H}), 7.01(\mathrm{~s}, 1 \mathrm{H}), 3.98(\mathrm{~s}$, $2 \mathrm{H}), 2.98(\mathrm{t}, J=7.2 \mathrm{~Hz}, 2 \mathrm{H}), 2.41(\mathrm{t}, J=6.8 \mathrm{~Hz}, 2 \mathrm{H}), 2.38$ $(\mathrm{s}, 6 \mathrm{H}), 2.02 \sim 1.94(\mathrm{~m}, 2 \mathrm{H}) ;{ }^{13} \mathrm{C}$ NMR $\left(100 \mathrm{MHz}, \mathrm{CDCl}_{3}\right)$ $\delta$ : $139.3,138.2,135.4,134.0,131.9,129.5,128.0,127.0$, $126.2,125.4,123.7,118.3,117.2,116.3,111.0,53.4,29.4$, 21.4, 19.5, 15.9; HRMS (ESI-TOF) calcd for $\mathrm{C}_{23} \mathrm{H}_{23} \mathrm{~N}_{2} \mathrm{O}_{2} \mathrm{~S}$ $(\mathrm{M}+\mathrm{H})^{+}$391.1475, found 391.1480.

4-((1-(Thiophen-2-yl)-9H-pyrrolo[1,2-a]indol-2-yl)sulfonyl)butanenitrile (3qa): $45.6 \mathrm{mg}, 62 \%$ yield. Gray solid, m.p. $155.3 \sim 155.8{ }^{\circ} \mathrm{C} ;{ }^{1} \mathrm{H}$ NMR (400 $\mathrm{MHz}, \mathrm{CDCl}_{3}$ ) $\delta: 7.85(\mathrm{~s}, 1 \mathrm{H}), 7.65 \sim 7.64(\mathrm{~m}, 1 \mathrm{H}), 7.51(\mathrm{~d}, J=7.2 \mathrm{~Hz}$, $1 \mathrm{H}), 7.43(\mathrm{t}, J=3.6 \mathrm{~Hz}, 2 \mathrm{H}), 7.36 \sim 7.34(\mathrm{~m}, 1 \mathrm{H}), 7.31 \sim$ $7.27(\mathrm{~m}, 1 \mathrm{H}), 7.14 \sim 7.12(\mathrm{~m}, 1 \mathrm{H}), 4.08(\mathrm{~s}, 2 \mathrm{H}), 3.18(\mathrm{t}$, $J=7.2 \mathrm{~Hz}, 2 \mathrm{H}), 2.51(\mathrm{t}, J=7.2 \mathrm{~Hz}, 2 \mathrm{H}), 2.12 \sim 2.05(\mathrm{~m}$, $2 \mathrm{H}) ;{ }^{13} \mathrm{C}$ NMR $\left(100 \mathrm{MHz}, \mathrm{CDCl}_{3}\right) \delta: 139.1,136.0,133.9$, $133.2,128.2,128.0,127.3,126.3,125.7,124.9,123.0$, $118.3,117.1,111.1,110.2,53.0,30.3,19.4,16.1$; HRMS (ESI-TOF) calcd for $\mathrm{C}_{19} \mathrm{H}_{17} \mathrm{~N}_{2} \mathrm{O}_{2} \mathrm{~S}_{2} \quad(\mathrm{M}+\mathrm{H})^{+}$369.0726, found 369.0732 .

4-((1-Methyl-9H-pyrrolo[1,2-a]indol-2-yl)sulfonyl)butanenitrile (3ra): $34.2 \mathrm{mg}, 57 \%$ yield. Yellow solid, m.p. $140.0 \sim 140.6{ }^{\circ} \mathrm{C} ;{ }^{1} \mathrm{H}$ NMR $\left(400 \mathrm{MHz}, \mathrm{CDCl}_{3}\right) \delta: 7.66(\mathrm{~s}$, $1 \mathrm{H}), 7.47(\mathrm{~d}, J=7.6 \mathrm{~Hz}, 1 \mathrm{H}), 7.40 \sim 7.35(\mathrm{~m}, 2 \mathrm{H}), 7.25 \sim$ $7.21(\mathrm{~m}, 1 \mathrm{H}), 3.83(\mathrm{~s}, 2 \mathrm{H}), 3.26(\mathrm{t}, J=7.2 \mathrm{~Hz}, 2 \mathrm{H}), 2.61(\mathrm{t}$, $J=7.2 \mathrm{~Hz}, 2 \mathrm{H}), 2.31(\mathrm{t}, J=0.8 \mathrm{~Hz}, 3 \mathrm{H}), 2.22 \sim 2.15(\mathrm{~m}$, $2 \mathrm{H}) ;{ }^{13} \mathrm{C} \mathrm{NMR}\left(100 \mathrm{MHz}, \mathrm{CDCl}_{3}\right) \delta: 139.4,134.9,134.4$, $128.0,126.3,125.2,123.5,118.4,115.1,111.4,110.8$, 54.7, 28.2, 19.5, 16.2, 9.8; HRMS (ESI-TOF) calcd for $\mathrm{C}_{16} \mathrm{H}_{17} \mathrm{~N}_{2} \mathrm{O}_{2} \mathrm{~S}(\mathrm{M}+\mathrm{H})^{+} 301.1005$, found 301.1010.

4-((9-Methyl-1-phenyl-9H-pyrrolo[1,2-a]indol-2-yl)sulfonyl)butanenitrile (3ta): $61.7 \mathrm{mg}, 82 \%$ yield. Yellow solid, m.p. $120.3 \sim 120.6{ }^{\circ} \mathrm{C} ;{ }^{1} \mathrm{H}$ NMR (400 MHz, $\mathrm{CDCl}_{3}$ ) $\delta: 7.80(\mathrm{~s}, 1 \mathrm{H}), 7.63 \sim 7.61(\mathrm{~m}, 2 \mathrm{H}), 7.47 \sim 7.37(\mathrm{~m}, 6 \mathrm{H})$, $7.30 \sim 7.25(\mathrm{~m}, 1 \mathrm{H}), 4.31 \sim 4.26(\mathrm{~m}, 1 \mathrm{H}), 2.93(\mathrm{t}, J=7.2$ $\mathrm{Hz}, 2 \mathrm{H}), 2.48 \sim 2.33(\mathrm{~m}, 2 \mathrm{H}), 2.05 \sim 1.91(\mathrm{~m}, 2 \mathrm{H}), 1.31(\mathrm{~d}$, $J=7.2 \mathrm{~Hz}, 3 \mathrm{H}) ;{ }^{13} \mathrm{C} \mathrm{NMR}\left(100 \mathrm{MHz}, \mathrm{CDCl}_{3}\right) \delta: 140.6$, $140.1,138.3,131.6,129.8,128.5,128.1,127.9,125.7$, $125.1,123.9,118.3,117.2,115.7,110.9,53.6,36.3,19.4$, 17.2, 15.9; HRMS (ESI-TOF) calcd for $\mathrm{C}_{22} \mathrm{H}_{21} \mathrm{~N}_{2} \mathrm{O}_{2} \mathrm{~S}$ $(\mathrm{M}+\mathrm{H})^{+}$377.1318, found 377.1323.

4-((8-Methyl-1-phenyl-9H-pyrrolo[1,2-a]indol-2-yl)sulfonyl)butanenitrile (3ua): $60.2 \mathrm{mg}, 80 \%$ yield. Yellow solid, m.p. 168.2 168.6 ${ }^{\circ} \mathrm{C} ;{ }^{1} \mathrm{H}$ NMR (400 MHz, $\mathrm{CDCl}_{3}$ ) $\delta: 7.84(\mathrm{~s}, 1 \mathrm{H}), 7.69 \sim 7.67(\mathrm{~m}, 2 \mathrm{H}), 7.48 \sim 7.45(\mathrm{~m}, 2 \mathrm{H})$, 
$7.40 \sim 7.36(\mathrm{~m}, 1 \mathrm{H}), 7.32(\mathrm{t}, J=7.6 \mathrm{~Hz}, 1 \mathrm{H}), 7.27 \sim 7.25$ $(\mathrm{m}, 1 \mathrm{H}), 7.08(\mathrm{~d}, J=7.6 \mathrm{~Hz}, 1 \mathrm{H}), 3.87(\mathrm{~s}, 2 \mathrm{H}), 2.97(\mathrm{t}, J=$ $7.2 \mathrm{~Hz}, 2 \mathrm{H}), 2.41(\mathrm{t}, J=7.6 \mathrm{~Hz}, 2 \mathrm{H}), 2.36(\mathrm{~s}, 3 \mathrm{H}), 2.01 \sim$ $1.94(\mathrm{~m}, 2 \mathrm{H}) ;{ }^{13} \mathrm{C} \mathrm{NMR}\left(100 \mathrm{MHz}, \mathrm{CDCl}_{3}\right) \delta$ : 138.9, $136.0,135.5,132.8,132.2,129.4,128.7,128.1,127.7$, $126.6,123.4,118.3,116.9,116.5,108.4,53.6,28.4,19.4$, 18.5, 15.9; HRMS (ESI-TOF) calcd for $\mathrm{C}_{22} \mathrm{H}_{21} \mathrm{~N}_{2} \mathrm{O}_{2} \mathrm{~S}$ $(\mathrm{M}+\mathrm{H})^{+}$377.1318, found 377.1323.

4-((8-Chloro-1-phenyl-9H-pyrrolo[1,2-a]indol-2-yl)sulfonyl)butanenitrile (3va): $60.2 \mathrm{mg}, 76 \%$ yield. Yellow solid, m.p. 170.3 170.8 ${ }^{\circ} \mathrm{C}$; ${ }^{1} \mathrm{H}$ NMR (400 MHz, $\mathrm{CDCl}_{3}$ ) $\delta: 7.86(\mathrm{~s}, 1 \mathrm{H}), 7.67(\mathrm{t}, J=7.2 \mathrm{~Hz}, 2 \mathrm{H}), 7.47(\mathrm{t}, J=7.6 \mathrm{~Hz}$, $2 \mathrm{H}), 7.41 \sim 7.33(\mathrm{~m}, 3 \mathrm{H}), 7.25(\mathrm{t}, J=8.0 \mathrm{~Hz}, 1 \mathrm{H}), 4.00(\mathrm{~s}$, $2 \mathrm{H}), 2.97(\mathrm{t}, J=7.2 \mathrm{~Hz}, 2 \mathrm{H}), 2.42(\mathrm{t}, J=7.2 \mathrm{~Hz}, 2 \mathrm{H})$, $2.02 \sim 1.94(\mathrm{~m}, 2 \mathrm{H}) ;{ }^{13} \mathrm{C}$ NMR $\left(100 \mathrm{MHz}, \mathrm{CDCl}_{3}\right) \delta$ : $140.3,134.8,132.6,131.9,131.6,129.6,129.3,128.8$, $128.0,125.7,124.4,118.2,117.4,116.8,109.3,53.5,29.2$, 19.3, 16.0; HRMS (ESI-TOF) calcd for $\mathrm{C}_{21} \mathrm{H}_{18}{ }^{35} \mathrm{ClN}_{2} \mathrm{O}_{2} \mathrm{~S}$ $(\mathrm{M}+\mathrm{H})^{+}$397.0772, found 397.0778.

4-((7-Methoxy-1-phenyl-9H-pyrrolo[1,2-a]indol-2-yl)sulfonyl)butanenitrile (3wa): $64.3 \mathrm{mg}, 82 \%$ yield. Yellow solid, m.p. 131.2 131.8 ${ }^{\circ} \mathrm{C}$; ${ }^{1} \mathrm{H}$ NMR (400 MHz, $\mathrm{CDCl}_{3}$ ) $\delta: 7.79(\mathrm{~s}, 1 \mathrm{H}), 7.66(\mathrm{t}, J=6.8 \mathrm{~Hz}, 2 \mathrm{H}), 7.47 \sim 7.43(\mathrm{~m}$, $2 \mathrm{H}), 7.38 \sim 7.31(\mathrm{~m}, 2 \mathrm{H}), 7.03(\mathrm{~d}, J=2.4 \mathrm{~Hz}, 1 \mathrm{H}), 6.94 \sim$ $6.91(\mathrm{~m}, 1 \mathrm{H}), 3.95(\mathrm{~s}, 2 \mathrm{H}), 3.85(\mathrm{~s}, 3 \mathrm{H}), 2.96(\mathrm{t}, J=7.2 \mathrm{~Hz}$, $2 \mathrm{H}), 2.41(\mathrm{t}, J=7.2 \mathrm{~Hz}, 2 \mathrm{H}), 2.01 \sim 1.93(\mathrm{~m}, 2 \mathrm{H}) ;{ }^{13} \mathrm{C}$ NMR $\left(100 \mathrm{MHz}, \mathrm{CDCl}_{3}\right) \delta: 157.9,135.6,135.4,133.0$, $132.1,129.3,128.7,127.7,122.8,118.3,117.0,116.2$, 112.8, 112.6, 111.4, 55.8, 53.6, 29.6, 19.4, 15.9; HRMS (ESI-TOF) calcd for $\mathrm{C}_{22} \mathrm{H}_{21} \mathrm{~N}_{2} \mathrm{O}_{3} \mathrm{~S} \quad(\mathrm{M}+\mathrm{H})^{+}$393.1267, found 393.1273 .

4-((7-Bromo-1-phenyl-9H-pyrrolo[1,2-a]indol-2-yl)sulfonyl)butanenitrile (3xa): $63.4 \mathrm{mg}, 72 \%$ yield. Yellow solid, m.p. $141.1 \sim 141.6{ }^{\circ} \mathrm{C}$; ${ }^{1} \mathrm{H}$ NMR $\left(400 \mathrm{MHz}, \mathrm{CDCl}_{3}\right)$ $\delta: 7.84(\mathrm{~s}, 1 \mathrm{H}), 7.65(\mathrm{t}, J=7.2 \mathrm{~Hz}, 2 \mathrm{H}), 7.60(\mathrm{~s}, 1 \mathrm{H})$, $7.56 \sim 7.53(\mathrm{~m}, 1 \mathrm{H}), 7.48 \sim 7.44(\mathrm{~m}, 2 \mathrm{H}), 7.40 \sim 7.36(\mathrm{~m}$, $1 \mathrm{H}), 7.31(\mathrm{~d}, J=8.4 \mathrm{~Hz}, 1 \mathrm{H}), 3.98(\mathrm{~s}, 2 \mathrm{H}), 2.96(\mathrm{t}, J=7.2$ $\mathrm{Hz}, 2 \mathrm{H}), 2.41(\mathrm{t}, J=7.2 \mathrm{~Hz}, 2 \mathrm{H}), 2.01 \sim 1.94(\mathrm{~m}, 2 \mathrm{H}) ;{ }^{13} \mathrm{C}$ NMR $\left(100 \mathrm{MHz}, \mathrm{CDCl}_{3}\right) \delta: 138.4,136.1,135.1,131.7$, $131.1,129.5,129.3,128.8,127.9,124.3,118.6,118.2$, 117.3, 116.6, 112.3, 53.5, 29.2, 19.3, 15.9; HRMS (ESI-TOF) calcd for $\mathrm{C}_{21} \mathrm{H}_{18}{ }^{79} \mathrm{BrN}_{2} \mathrm{O}_{2} \mathrm{~S} \quad(\mathrm{M}+\mathrm{H})^{+}$ 441.0267, found 441.0273.

4-((6-Methyl-1-phenyl-9H-pyrrolo[1,2-a]indol-2-yl)sulfonyl)butanenitrile (3ya): $62.4 \mathrm{mg}, 83 \%$ yield. Yellow solid, m.p. 125.5 126.1 ${ }^{\circ} \mathrm{C}$; ${ }^{1} \mathrm{H}$ NMR (400 MHz, $\mathrm{CDCl}_{3}$ ) $\delta: 7.84(\mathrm{~s}, 1 \mathrm{H}), 7.68 \sim 7.66(\mathrm{~m}, 2 \mathrm{H}), 7.47 \sim 7.43(\mathrm{~m}, 2 \mathrm{H})$, $7.39 \sim 7.33(\mathrm{~m}, 2 \mathrm{H}), 7.25(\mathrm{~s}, 1 \mathrm{H}), 7.07(\mathrm{~d}, J=7.6 \mathrm{~Hz}$, $1 \mathrm{H}), 3.93(\mathrm{~s}, 2 \mathrm{H}), 2.97(\mathrm{t}, J=7.2 \mathrm{~Hz}, 2 \mathrm{H}), 2.46(\mathrm{~s}, 3 \mathrm{H})$, $2.41(\mathrm{t}, J=7.2 \mathrm{~Hz}, 2 \mathrm{H}), 2.01 \sim 1.94(\mathrm{~m}, 2 \mathrm{H}) ;{ }^{13} \mathrm{C} \mathrm{NMR}$ $\left(100 \mathrm{MHz}, \mathrm{CDCl}_{3}\right) \delta: 139.4,138.4,136.0,132.1,130.9$, $129.3,128.7,127.7,126.3,125.8,123.4,118.3,116.9$, $116.4,111.7,53.6,29.1,21.6,19.4,15.9$; HRMS (ESI-TOF) calcd for $\mathrm{C}_{22} \mathrm{H}_{21} \mathrm{~N}_{2} \mathrm{O}_{2} \mathrm{~S}(\mathrm{M}+\mathrm{H})^{+} 377.1318$, found 377.1323 .

4-((6-Fluoro-1-phenyl-9H-pyrrolo[1,2-a]indol-2-yl)sulfonyl)butanenitrile (3za): $59.3 \mathrm{mg}, 78 \%$ yield. Yellow solid, m.p. 113.4 113.9 ${ }^{\circ} \mathrm{C} ;{ }^{1} \mathrm{H}$ NMR (400 MHz, $\mathrm{CDCl}_{3}$ ) $\delta: 7.83(\mathrm{~s}, 1 \mathrm{H}), 7.67 \sim 7.64(\mathrm{~m}, 2 \mathrm{H}), 7.48 \sim 7.46(\mathrm{~m}, 2 \mathrm{H})$, $7.44 \sim 7.38(\mathrm{~m}, 2 \mathrm{H}), 7.17 \sim 7.14(\mathrm{~m}, 1 \mathrm{H}), 6.99 \sim 6.94(\mathrm{~m}$, $1 \mathrm{H}), 3.95(\mathrm{~s}, 2 \mathrm{H}), 2.97(\mathrm{t}, J=7.2 \mathrm{~Hz}, 2 \mathrm{H}), 2.41(\mathrm{t}, J=7.2$ $\mathrm{Hz}, 2 \mathrm{H}), 2.01 \sim 1.94(\mathrm{~m}, 2 \mathrm{H}) ;{ }^{13} \mathrm{C} \mathrm{NMR}(100 \mathrm{MHz}$, $\left.\mathrm{CDCl}_{3}\right) \delta: 162.6(\mathrm{~d}, J=245.3 \mathrm{~Hz}, 1 \mathrm{C}), 140.2(\mathrm{~d}, J=11.3$ $\mathrm{Hz}, 1 \mathrm{C}), 136.4,131.7,129.3(\mathrm{~d}, J=2.2 \mathrm{~Hz}, 1 \mathrm{C}), 128.8$, $127.1(\mathrm{~d}, J=9.5 \mathrm{~Hz}, 1 \mathrm{C}), 124.4,118.2,117.2,116.6$, $112.3,112.1,99.8,99.5,53.5,28.8,19.3,15.9 ;{ }^{19} \mathrm{~F}$ NMR $\left(282 \mathrm{MHz}, \mathrm{CDCl}_{3}\right) \delta$ : $-112.1(\mathrm{~s}, 1 \mathrm{~F})$; HRMS (ESI-TOF) calcd for $\mathrm{C}_{21} \mathrm{H}_{18}{ }^{19} \mathrm{FN}_{2} \mathrm{O}_{2} \mathrm{~S}(\mathrm{M}+\mathrm{H})^{+}$381.1068, found 381.1073 .

4-((5-Methyl-1-phenyl-9H-pyrrolo[1,2-a]indol-2-yl)sulfonyl)butanenitrile (3aaa): $60.2 \mathrm{mg}, 80 \%$ yield. Yellow solid, m.p. $125.0 \sim 125.6{ }^{\circ} \mathrm{C} ;{ }^{1} \mathrm{H}$ NMR (400 MHz, $\mathrm{CDCl}_{3}$ ) $\delta: 7.96(\mathrm{~s}, 1 \mathrm{H}), 7.68 \sim 7.66(\mathrm{~m}, 2 \mathrm{H}), 7.48 \sim 7.44(\mathrm{~m}, 2 \mathrm{H})$, $7.39 \sim 7.36(\mathrm{~m}, 1 \mathrm{H}), 7.31(\mathrm{~d}, J=6.8 \mathrm{~Hz}, 1 \mathrm{H}), 7.20 \sim 7.14$ $(\mathrm{m}, 2 \mathrm{H}), 3.97(\mathrm{~s}, 2 \mathrm{H}), 2.97(\mathrm{t}, J=7.2 \mathrm{~Hz}, 2 \mathrm{H}), 2.66(\mathrm{~s}, 3 \mathrm{H})$, $2.41(\mathrm{t}, J=7.2 \mathrm{~Hz}, 2 \mathrm{H}), 2.02 \sim 1.95(\mathrm{~m}, 2 \mathrm{H}) ;{ }^{13} \mathrm{C} \mathrm{NMR}$ $\left(100 \mathrm{MHz}, \mathrm{CDCl}_{3}\right) \delta: 138.2,136.0,134.1,132.1,130.3$, $129.4,128.7,127.7,125.4,123.7,123.5,123.0,119.1$, $118.3, \quad 116.5, \quad 53.6, \quad 29.2,19.4,18.4,16.0$; HRMS (ESI-TOF) calcd for $\mathrm{C}_{22} \mathrm{H}_{21} \mathrm{~N}_{2} \mathrm{O}_{2} \mathrm{~S}(\mathrm{M}+\mathrm{H})^{+}$377.1318, found 377.1323 .

5-Phenyl-4-((1-phenyl-9H-pyrrolo[1,2-a]indol-2-yl)sulfonyl)pentanenitrile (3ab): $59.7 \mathrm{mg}, 66 \%$ yield, brown oil. ${ }^{1} \mathrm{H}$ NMR $\left(400 \mathrm{MHz}, \mathrm{CDCl}_{3}\right) \delta: 7.86(\mathrm{~s}, 1 \mathrm{H}), 7.72(\mathrm{t}$, $J=7.2 \mathrm{~Hz}, 2 \mathrm{H}), 7.52 \sim 7.47(\mathrm{~m}, 3 \mathrm{H}), 7.43 \sim 7.39(\mathrm{~m}, 3 \mathrm{H})$, $7.29 \sim 7.27(\mathrm{~m}, 1 \mathrm{H}), 7.20 \sim 7.14(\mathrm{~m}, 3 \mathrm{H}), 6.82(\mathrm{~d}, J=6.4$ $\mathrm{Hz}, 2 \mathrm{H}), 4.07 \sim 3.92$ (m. 2H), 3.18 (d, J=3.6 Hz, 1H), $3.15(\mathrm{~d}, J=3.6 \mathrm{~Hz}, 1 \mathrm{H}), 2.54 \sim 2.48(\mathrm{~m}, 1 \mathrm{H}), 2.33 \sim 2.27$ $(\mathrm{m}, 2 \mathrm{H}), 2.12 \sim 2.04(\mathrm{~m}, 1 \mathrm{H}), 1.80 \sim 1.77(\mathrm{~m}, 1 \mathrm{H}) ;{ }^{13} \mathrm{C}$ NMR $\left(100 \mathrm{MHz}, \mathrm{CDCl}_{3}\right) \delta: 139.3,136.2,135.6,134.0$, $132.3,129.5,128.8,128.8,128.7,128.1,128.0,127.0$, 126.3, 125.6, 122.4, 118.6, 117.2, 117.1, 111.0, 62.8, 34.9, 29.4, 23.2, 15.3; HRMS (ESI-TOF) calcd for $\mathrm{C}_{28} \mathrm{H}_{25} \mathrm{~N}_{2} \mathrm{O}_{2} \mathrm{~S}$ $(\mathrm{M}+\mathrm{H})^{+}$453.1631, found 453.1636 .

3-Phenyl-4-((1-phenyl-9H-pyrrolo[1,2-a]indol-2-yl)sulfonyl)butanenitrile (3ac): $68.3 \mathrm{mg}, 78 \%$ yield, yellow oil. ${ }^{1} \mathrm{H}$ NMR (400 MHz, $\left.\mathrm{CDCl}_{3}\right) \delta: 7.74(\mathrm{~s}, 1 \mathrm{H}), 7.64 \sim$ $7.61(\mathrm{~m}, 2 \mathrm{H}), 7.47 \sim 7.35(\mathrm{~m}, 6 \mathrm{H}), 7.33 \sim 7.24(\mathrm{~m}, 1 \mathrm{H})$, $7.21 \sim 7.17(\mathrm{~m}, 2 \mathrm{H}), 7.15 \sim 7.11(\mathrm{~m}, 1 \mathrm{H}), 6.98 \sim 6.95(\mathrm{~m}$, $2 \mathrm{H}), 3.98 \sim 3.86(\mathrm{~m}, 2 \mathrm{H}), 3.49 \sim 3.43(\mathrm{~m}, 1 \mathrm{H}), 3.33 \sim 3.18$ $(\mathrm{m}, 2 \mathrm{H}), 2.85 \sim 2.69(\mathrm{~m}, 2 \mathrm{H}) ;{ }^{13} \mathrm{C} \mathrm{NMR}(100 \mathrm{MHz}$, $\left.\mathrm{CDCl}_{3}\right) \delta: 139.2,139.0,135.4,133.9,132.0,129.3,128.7$, $128.7,128.1,127.8,127.7,126.9,126.2,125.5,123.8$, $117.3,116.9,116.8,111.0,59.0,36.9,29.3,24.0$; HRMS (ESI-TOF) calcd for $\mathrm{C}_{27} \mathrm{H}_{23} \mathrm{~N}_{2} \mathrm{O}_{2} \mathrm{~S}(\mathrm{M}+\mathrm{H})^{+}$439.1475, found 439.1480 .

4-((1-Phenyl-9H-pyrrolo[1,2-a]indol-2-yl)sulfonyl)-3(p-tolyl)butanenitrile (3ad): $65.1 \mathrm{mg}, 72 \%$ yield, yellow oil. ${ }^{1} \mathrm{H}$ NMR (400 MHz, $\left.\mathrm{CDCl}_{3}\right) \delta: 7.67(\mathrm{~s}, 1 \mathrm{H}), 7.65 \sim$ $7.63(\mathrm{~m}, 2 \mathrm{H}), 7.47 \sim 7.44(\mathrm{~m}, 4 \mathrm{H}), 7.43 \sim 7.36(\mathrm{~m}, 2 \mathrm{H})$, $7.28 \sim 7.25(\mathrm{~m}, 1 \mathrm{H}), 6.97(\mathrm{~d}, J=8.0 \mathrm{~Hz}, 2 \mathrm{H}), 6.85(\mathrm{~d}, J=$ $8.0 \mathrm{~Hz}, 2 \mathrm{H}), 3.98 \sim 3.86(\mathrm{~m}, 2 \mathrm{H}), 3.49 \sim 3.40(\mathrm{~m}, 1 \mathrm{H})$, $3.30 \sim 3.16(\mathrm{~m}, 2 \mathrm{H}), 2.80 \sim 2.64(\mathrm{~m}, 2 \mathrm{H}), 2.10(\mathrm{~s}, 3 \mathrm{H}) ;{ }^{13} \mathrm{C}$ NMR $\left(100 \mathrm{MHz}, \mathrm{CDCl}_{3}\right) \delta: 139.2,137.6,135.8,135.3$, 
$133.9,132.0,129.3,129.3,128.7,128.1,127.7,126.9$, 126.2, 125.5, 123.8, 117.4, 117.0, 116.8, 111.0, 59.0, 36.7, 29.3, 24.2, 20.8; HRMS (ESI-TOF) calcd for $\mathrm{C}_{28} \mathrm{H}_{25} \mathrm{~N}_{2} \mathrm{O}_{2} \mathrm{~S}$ $(\mathrm{M}+\mathrm{H})^{+}$453.1631, found 453.1637 .

3-(4-(tert-Butyl)phenyl)-4-((1-phenyl-9H-pyrrolo[1,2$a$ ]indol-2-yl)sulfonyl)butanenitrile (3ae): $74.1 \mathrm{mg}, 75 \%$ yield, brown oil. ${ }^{1} \mathrm{H}$ NMR $\left(400 \mathrm{MHz}, \mathrm{CDCl}_{3}\right) \delta: 7.76(\mathrm{~s}$, $1 \mathrm{H}), 7.63 \sim 7.61(\mathrm{~m}, 2 \mathrm{H}), 7.45 \sim 7.34(\mathrm{~m}, 6 \mathrm{H}), 7.27 \sim 7.23$ $(\mathrm{m}, 1 \mathrm{H}), 7.21 \sim 7.19(\mathrm{~m}, 2 \mathrm{H}), 6.89(\mathrm{~d}, J=8.4 \mathrm{~Hz}, 2 \mathrm{H})$, $3.97 \sim 3.85(\mathrm{~m}, 2 \mathrm{H}), 3.50 \sim 3.41(\mathrm{~m}, 1 \mathrm{H}), 3.33 \sim 3.19(\mathrm{~m}$, $2 \mathrm{H}), 2.82 \sim 2.68(\mathrm{~m}, 2 \mathrm{H}), 1.18(\mathrm{~s}, 9 \mathrm{H}) ;{ }^{13} \mathrm{C} \mathrm{NMR}(100$ $\left.\mathrm{MHz}, \mathrm{CDCl}_{3}\right) \delta: 150.8,139.2,136.0,135.3,133.9,132.0$, $129.4,128.7,128.1,127.7,126.6,126.2,125.6,125.5$, $123.9,117.5,116.9,116.7,110.9,59.0,36.4,34.3,31.1$, 29.3, 24.1; HRMS (ESI-TOF) calcd for $\mathrm{C}_{31} \mathrm{H}_{31} \mathrm{~N}_{2} \mathrm{O}_{2} \mathrm{~S}$ $(\mathrm{M}+\mathrm{H})^{+}$495.2101, found 495.2106.

3-(4-Fluorophenyl)-4-((1-phenyl-9H-pyrrolo[1,2a]indol-2-yl)sulfonyl)butanenitrile (3af): $63.8 \mathrm{mg}, 70 \%$ yield, yellow oil. ${ }^{1} \mathrm{H}$ NMR $\left(400 \mathrm{MHz}, \mathrm{CDCl}_{3}\right) \delta$ : $7.78(\mathrm{~s}$, $1 \mathrm{H}), 7.63 \sim 7.61(\mathrm{~m}, 2 \mathrm{H}), 7.48 \sim 7.44(\mathrm{~m}, 5 \mathrm{H}), 7.42 \sim 7.34$ $(\mathrm{m}, 1 \mathrm{H}), 7.29 \sim 7.25(\mathrm{~m}, 1 \mathrm{H}), 7.00 \sim 6.87(\mathrm{~m}, 4 \mathrm{H}), 4.01 \sim$ $3.89(\mathrm{~m}, 2 \mathrm{H}), 3.49 \sim 3.42(\mathrm{~m}, 1 \mathrm{H}), 3.30 \sim 3.14(\mathrm{~m}, 2 \mathrm{H})$, $2.84 \sim 2.67(\mathrm{~m}, 2 \mathrm{H}) ;{ }^{13} \mathrm{C} \mathrm{NMR}\left(100 \mathrm{MHz}, \mathrm{CDCl}_{3}\right) \delta: 162.1$ (d, $J=245.9 \mathrm{~Hz}, 1 \mathrm{C}), 139.1,135.5,134.9,133.9,131.9$, 129.3, 128.7 (t, $J=4.8 \mathrm{~Hz}, 1 \mathrm{C}), 128.1,127.8,126.3,125.6$, 123.8, 117.1, 116.8, 116.7, 115.8, 115.6, 111.0, 59.0, 36.2, 29.3, 24.2; ${ }^{19} \mathrm{~F}$ NMR (282 MHz, $\left.\mathrm{CDCl}_{3}\right) \delta$ : $-113.9(\mathrm{~s}$, $1 \mathrm{~F}$ ); HRMS (ESI-TOF) calcd for $\mathrm{C}_{27} \mathrm{H}_{22}{ }^{19} \mathrm{FN}_{2} \mathrm{O}_{2} \mathrm{~S}$ (M+ H) ${ }^{+}$457.1381, found 457.1387.

3-(4-Bromophenyl)-4-((1-phenyl-9H-pyrrolo[1,2a]indol-2-yl)sulfonyl)butanenitrile (3ag): $68.1 \mathrm{mg}, 66 \%$ yield, yellow oil. ${ }^{1} \mathrm{H}$ NMR $\left(400 \mathrm{MHz}, \mathrm{CDCl}_{3}\right) \delta: 7.73(\mathrm{~s}$, $1 \mathrm{H}), 7.63 \sim 7.60(\mathrm{~m}, 2 \mathrm{H}), 7.48 \sim 7.36(\mathrm{~m}, 6 \mathrm{H}), 7.30 \sim 7.26$ $(\mathrm{m}, 3 \mathrm{H}), 6.83(\mathrm{~d}, J=8.4 \mathrm{~Hz}, 2 \mathrm{H}), 4.00 \sim 3.87(\mathrm{~m}, 2 \mathrm{H})$, $3.49 \sim 3.40(\mathrm{~m}, 1 \mathrm{H}), 3.28 \sim 3.14(\mathrm{~m}, 2 \mathrm{H}), 2.80 \sim 2.65(\mathrm{~m}$, $2 \mathrm{H}) ;{ }^{13} \mathrm{C} \mathrm{NMR}\left(100 \mathrm{MHz}, \mathrm{CDCl}_{3}\right) \delta: 139.0,137.8,135.5$, $133.9,131.9,131.8,129.2,128.7(2 \mathrm{C}), 128.1,127.8,126.3$, 125.6, 123.6, 121.8, 117.0, 116.9, 116.7, 111.0, 58.6, 36.6, 29.4, 24.0; HRMS (ESI-TOF) calcd for $\mathrm{C}_{27} \mathrm{H}_{22}{ }^{79} \mathrm{BrN}_{2} \mathrm{O}_{2} \mathrm{~S}$ $(\mathrm{M}+\mathrm{H})^{+} 517.0580$, found 517.0585 .

3-Benzyl-4-((1-phenyl-9H-pyrrolo[1,2-a]indol-2-yl)sulfonyl)butanenitrile (3ah): $64.2 \mathrm{mg}, 71 \%$ yield, yellow oil. ${ }^{1} \mathrm{H}$ NMR $\left(400 \mathrm{MHz}, \mathrm{CDCl}_{3}\right) \delta: 7.85(\mathrm{~s}, 1 \mathrm{H}), 7.61 \sim 759$ $(\mathrm{m}, 2 \mathrm{H}), 7.48 \sim 7.42(\mathrm{~m}, 5 \mathrm{H}), 7.41 \sim 7.37(\mathrm{~m}, 1 \mathrm{H}), 7.36 \sim$ $7.25(\mathrm{~m}, 1 \mathrm{H}), 7.24 \sim 7.20(\mathrm{~m}, 3 \mathrm{H}), 7.02 \sim 7.00(\mathrm{~m}, 2 \mathrm{H})$, $4.00(\mathrm{~s}, 2 \mathrm{H}), 2.97(\mathrm{~d}, J=7.2 \mathrm{~Hz}, 2 \mathrm{H}), 2.69 \sim 2.61(\mathrm{~m}, 2 \mathrm{H})$, $2.59 \sim 2.55(\mathrm{~m}, 1 \mathrm{H}), 2.54 \sim 2.51(\mathrm{~m}, 1 \mathrm{H}), 2.40 \sim 2.35(\mathrm{~m}$, $1 \mathrm{H}) ;{ }^{13} \mathrm{C}$ NMR $\left(100 \mathrm{MHz}, \mathrm{CDCl}_{3}\right) \delta: 139.3,136.7,135.5$, $134.0,132.0,129.3,128.9,128.7,128.7,128.1,127.7$, $127.0,126.3,125.6,124.3,117.4,117.1,116.3,111.0$, 57.5, 39.3, 32.2, 29.4, 20.8; HRMS (ESI-TOF) calcd for $\mathrm{C}_{28} \mathrm{H}_{25} \mathrm{~N}_{2} \mathrm{O}_{2} \mathrm{~S}(\mathrm{M}+\mathrm{H})^{+}$453.1631, found 453.1636.

3-(Benzyloxy)-4-((1-phenyl-9H-pyrrolo[1,2-a]indol-2yl)sulfonyl)butanenitrile (3ai): $73.9 \mathrm{mg}, 79 \%$ yield, yellow oil. ${ }^{1} \mathrm{H}$ NMR (400 MHz, $\left.\mathrm{CDCl}_{3}\right) \delta: 7.83(\mathrm{~s}, 1 \mathrm{H}), 7.65 \sim$ $7.63(\mathrm{~m}, 2 \mathrm{H}), 7.48 \sim 7.44(\mathrm{~m}, 3 \mathrm{H}), 7.43 \sim 7.38(\mathrm{~m}, 3 \mathrm{H})$, $7.30 \sim 7.26(\mathrm{~m}, 1 \mathrm{H}), 7.20 \sim 7.17(\mathrm{~m}, 3 \mathrm{H}), 7.13 \sim 7.11(\mathrm{~m}$,
$2 \mathrm{H}), 4.35 \sim 4.27(\mathrm{~m}, 2 \mathrm{H}), 4.04 \sim 3.98(\mathrm{~m}, 1 \mathrm{H}), 3.93(\mathrm{~s}$, $2 \mathrm{H}), 3.33 \sim 3.29(\mathrm{~m}, 1 \mathrm{H}), 3.11 \sim 3.06(\mathrm{~m}, 1 \mathrm{H}), 2.77 \sim 2.72$ $(\mathrm{m}, 1 \mathrm{H}), 2.61 \sim 2.55(\mathrm{~m}, 1 \mathrm{H}) ;{ }^{13} \mathrm{C} \mathrm{NMR}(100 \mathrm{MHz}$, $\left.\mathrm{CDCl}_{3}\right) \delta: 139.2,136.3,135.5,133.9,132.0,129.4,128.8$, $128.3,128.1,128.0,127.9,127.7,126.2,125.6,124.2$, $116.9,116.5,116.3,111.0,71.7,69.2,58.0,29.3,23.8$; HRMS (ESI-TOF) calcd for $\mathrm{C}_{28} \mathrm{H}_{25} \mathrm{~N}_{2} \mathrm{O}_{3} \mathrm{~S}(\mathrm{M}+\mathrm{H})$ 469.1580, found 469.1586 .

Benzyl 3-cyano-2-(((1-phenyl-9H-pyrrolo[1,2-a]indol2-yl)sulfonyl)methyl)propanoate (3aj): $74.4 \mathrm{mg}, \quad 75 \%$ yield, yellow oil. ${ }^{1} \mathrm{H}$ NMR $\left(400 \mathrm{MHz}, \mathrm{CDCl}_{3}\right) \delta: 7.85(\mathrm{~s}$, $1 \mathrm{H}), 7.64 \sim 7.61(\mathrm{~m}, 2 \mathrm{H}), 7.47(\mathrm{~d}, J=7.6 \mathrm{~Hz}, 1 \mathrm{H}), 7.43 \sim$ $7.40(\mathrm{~m}, 4 \mathrm{H}), 7.37 \sim 7.33(\mathrm{~m}, 4 \mathrm{H}), 7.29 \sim 7.24(\mathrm{~m}, 3 \mathrm{H})$, $5.12 \sim 5.03(\mathrm{~m}, 2 \mathrm{H}), 3.96(\mathrm{~s}, 2 \mathrm{H}), 3.46(\mathrm{~d}, J=10.4 \mathrm{~Hz}$, $1 \mathrm{H}), 3.15 \sim 3.09(\mathrm{~m}, 2 \mathrm{H}), 2.85 \sim 2.82(\mathrm{~m}, 2 \mathrm{H}) ;{ }^{13} \mathrm{C} \mathrm{NMR}$ $\left(100 \mathrm{MHz}, \mathrm{CDCl}_{3}\right) \delta: 169.4,139.2,135.7,134.6,134.0$, $131.7,129.4,128.7,128.6(2 \mathrm{C}), 128.4,128.1,127.9,126.3$, $125.7,123.4,117.2,116.7,116.6,111.1,68.0,54.7,37.0$, 29.3, 18.9; HRMS (ESI-TOF) calcd for $\mathrm{C}_{29} \mathrm{H}_{25} \mathrm{~N}_{2} \mathrm{O}_{4} \mathrm{~S}$ $(\mathrm{M}+\mathrm{H})^{+}$497.1530, found 497.1535.

Ethyl 3-cyano-2-(((1-phenyl-9H-pyrrolo[1,2-a]indol-2yl)sulfonyl)methyl)propanoate (3ak): $62.5 \mathrm{mg}, 72 \%$ yield. White solid, m.p. 148.2 148.6 ${ }^{\circ} \mathrm{C}$; ${ }^{1} \mathrm{H}$ NMR $(400 \mathrm{MHz}$, $\left.\mathrm{CDCl}_{3}\right) \delta: 7.88(\mathrm{~s}, 1 \mathrm{H}), 7.68 \sim 7.66(\mathrm{~m}, 2 \mathrm{H}), 7.49 \sim 7.38$ $(\mathrm{m}, 6 \mathrm{H}), 7.30 \sim 7.26(\mathrm{~m}, 1 \mathrm{H}), 4.16 \sim 4.07(\mathrm{~m}, 2 \mathrm{H}), 3.99(\mathrm{~s}$, $2 \mathrm{H}), 3.49 \sim 3.43(\mathrm{~m}, 1 \mathrm{H}), 3.14 \sim 3.07(\mathrm{~m}, 2 \mathrm{H}), 2.89 \sim 2.78$ $(\mathrm{m}, 2 \mathrm{H}), 1.19$ (t, J=7.2 Hz, 3H); ${ }^{13} \mathrm{C} \mathrm{NMR}(100 \mathrm{MHz}$, $\left.\mathrm{CDCl}_{3}\right) \delta: 169.5,139.2,135.7,134.0,131.8,129.4,128.7$, $128.1,127.8,126.3,125.7,123.5,117.1,116.7,116.7$, 111.1, 62.3, 54.7, 36.9, 29.4, 18.8, 13.9; HRMS (ESI-TOF) calcd for $\mathrm{C}_{24} \mathrm{H}_{23} \mathrm{~N}_{2} \mathrm{O}_{4} \mathrm{~S}(\mathrm{M}+\mathrm{H})^{+} 435.1373$, found 435.1378 .

4-(Naphthalen-1-yl)-3-(((1-phenyl-9H-pyrrolo[1,2- $a]$ indol-2-yl)sulfonyl)methyl)butanenitrile (3al): $66.3 \mathrm{mg}, 66 \%$ yield, brown oil. ${ }^{1} \mathrm{H}$ NMR (400 $\left.\mathrm{MHz}, \mathrm{CDCl}_{3}\right) \delta: 7.76 \sim$ $7.69(\mathrm{~m}, 4 \mathrm{H}), 7.48 \sim 7.26(\mathrm{~m}, 12 \mathrm{H}), 7.19(\mathrm{~d}, J=6.8 \mathrm{~Hz}$, $1 \mathrm{H}), 3.86(\mathrm{~s}, 2 \mathrm{H}), 3.14 \sim 3.00(\mathrm{~m}, 4 \mathrm{H}), 2.68 \sim 2.65(\mathrm{~m}$, $1 \mathrm{H}), 2.61 \sim 2.55(\mathrm{~m}, 1 \mathrm{H}), 2.44 \sim 2.39(\mathrm{~m}, 1 \mathrm{H}) ;{ }^{13} \mathrm{C} \mathrm{NMR}$ $\left(100 \mathrm{MHz}, \mathrm{CDCl}_{3}\right) \delta: 139.2,135.4,134.0,133.0,131.8$, $131.3,129.3,128.8,128.5(2 \mathrm{C}), 128.1,128.0,127.7,127.7$, $126.2,126.1,125.7,125.6,125.4,123.6,123.1,117.5$, 117.1, 116.4, 111.0, 57.9, 37.0, 31.7, 29.3, 21.5; HRMS (ESI-TOF) calcd for $\mathrm{C}_{32} \mathrm{H}_{27} \mathrm{~N}_{2} \mathrm{O}_{2} \mathrm{~S}(\mathrm{M}+\mathrm{H})^{+}$503.1788, found 503.1794.

3-Methyl-3-phenyl-4-((1-phenyl-9H-pyrrolo[1,2-a]indol-2-yl)sulfonyl)butanenitrile (3am): $54.2 \mathrm{mg}, 60 \%$ yield, yellow oil. ${ }^{1} \mathrm{H}$ NMR (400 MHz, $\left.\mathrm{CDCl}_{3}\right) \delta: 7.68(\mathrm{t}, J=7.2$ $\mathrm{Hz}, 2 \mathrm{H}), 7.51 \sim 7.44(\mathrm{~m}, 4 \mathrm{H}), 7.43 \sim 7.40(\mathrm{~m}, 2 \mathrm{H}), 7.31(\mathrm{~d}$, $J=7.6 \mathrm{~Hz}, 1 \mathrm{H}), 7.27 \sim 7.23(\mathrm{~m}, 1 \mathrm{H}), 7.18 \sim 7.14(\mathrm{~m}, 2 \mathrm{H})$, $7.04 \sim 7.01(\mathrm{~m}, 3 \mathrm{H}), 3.92(\mathrm{~s}, 2 \mathrm{H}), 3.26 \sim 3.15(\mathrm{~m}, 3 \mathrm{H})$, $2.96(\mathrm{~d}, J=16.8 \mathrm{~Hz}, 1 \mathrm{H}), 1.66(\mathrm{~s}, 3 \mathrm{H}) ;{ }^{13} \mathrm{C}$ NMR $(100$ $\left.\mathrm{MHz}, \mathrm{CDCl}_{3}\right) \delta: 141.5,139.2,135.0,133.8,132.2,129.4$, $128.7,128.2,128.1,127.7,127.2,126.2,125.6,125.5$, $125.4,117.4,116.5,116.4,110.9,64.3,40.0,29.3,29.0$, 25.7; HRMS (ESI-TOF) calcd for $\mathrm{C}_{28} \mathrm{H}_{25} \mathrm{~N}_{2} \mathrm{O}_{2} \mathrm{~S}(\mathrm{M}+\mathrm{H})^{+}$ 453.1631, found 453.1636.

2-(((1-Phenyl-9H-pyrrolo[1,2-a]indol-2-yl)sulfonyl)- 
methoxy)acetonitrile (3an): $32.8 \mathrm{mg}, 45 \%$ yield. Yellow solid, m.p. 151.2 151.6 ${ }^{\circ} \mathrm{C} ;{ }^{1} \mathrm{H}$ NMR (400 MHz, $\mathrm{CDCl}_{3}$ ) $\delta: 7.89(\mathrm{~s}, 1 \mathrm{H}), 7.63 \sim 7.61(\mathrm{~m}, 2 \mathrm{H}), 7.49 \sim 7.42(\mathrm{~m}, 5 \mathrm{H})$, $7.40 \sim 7.36(\mathrm{~m}, 1 \mathrm{H}), 7.29 \sim 7.25(\mathrm{~m}, 1 \mathrm{H}), 4.38(\mathrm{~s}, 2 \mathrm{H})$, 4.29 (s, 2H), 3.97 (s, 2H); $\left.{ }^{13} \mathrm{C} \mathrm{NMR} \mathrm{(100} \mathrm{MHz,} \mathrm{CDCl}_{3}\right) \delta$ : $139.2,135.8,134.1,132.1,129.7,128.5,128.1,127.8$, $126.3,125.7,121.2,118.0,117.6,114.7,111.1,84.0,56.5$, 29.2; HRMS (ESI-TOF) calcd for $\mathrm{C}_{20} \mathrm{H}_{17} \mathrm{~N}_{2} \mathrm{O}_{3} \mathrm{~S}(\mathrm{M}+\mathrm{H})^{+}$ 365.0954, found 365.0960.

\subsection{Gram scale experiment}

A gram scale experiment was carried out in the presence of $N$-propargylindoles $1 \mathrm{a}(1 \mathrm{~g}, 4.33 \mathrm{mmol})$, cyclobutanone oxime ester $2 \mathrm{a}$ (1.5 equiv., $6.5 \mathrm{mmol}), \mathrm{Na}_{2}$-Eosin $\mathrm{Y}(59.9$ $\mathrm{mg}, 0.0866 \mathrm{mmol}, 2 \mathrm{~mol} \%), \mathrm{K}_{2} \mathrm{~S}_{2} \mathrm{O}_{5}$ (1925.2 $\mathrm{mg}, 8.66$ mmol, 2 equiv.) and $\mathrm{MeCN}(40 \mathrm{~mL})$ at $80{ }^{\circ} \mathrm{C}$ for $64 \mathrm{~h}$. Cyanoalkylsulfonylated oxindole 3aa was obtained in 52\% yield $(815.3 \mathrm{mg})$.

Supporting Information The NMR spectra copies of products 3aa $\sim$ 3aaa and products 3ab $\sim$ 3am. The Supporting Information is available free of charge via the Internet at http://sioc-journal.cn.

\section{References}

[1] (a) Wang, T.; Jiao, N. Acc. Chem. Res. 2014, 47, 1137.

(b) Hu, P.; Chai, J.-C.; Duan, Y.-L.; Liu, Z.-H.; Cui, G.-L.; Chen, L.-Q. J. Mater. Chem. A 2016, 4, 10070.

(c) Bagal, D.-B.; Bhanage, B.-M. Adv. Synth. Catal. 2015, 357, 883. (d) Yan, G.; Zhang, Y.; Wang, J. Adv. Synth. Catal. 2017, 359, 4068.

(e) Fleming, F.-F.; Yao, L.-H.; Ravikumar, P.-C.; Funk, L.; Shook, B.-C. J. Med. Chem. 2010, 53, 7902.

(f) Rappoport, Z. The Chemistry of the Cyano Group, Wiley, London, 1970, pp. 121 312 .

(g) Fleming, F.-F. Nat. Prod. Rep. 1999, 16, 597.

(h) Fatiadi, A. J. In Preparation and Synthetic Applications of Cyano Compounds, Eds.: Patai, S.; Rappoport, Z., Wiley-VCH, New York, 1983.

[2] (a) Suto, Y.; Tsuji, R.; Kanai, M.; Shibasaki, M. Org. Lett. 2005, 7, 3757.

(b) Wang, G.-W.; Zhou, A.-X.; Wang, J.-J.; Hu, R.-B.; Yang, S.-D. Org. Lett. 2013, 15, 5270.

(c) Shen, J.-X.; Yang, D.-J.; Liu, Y.-X.; Qin, S.-S.; Zhang, J.-W.; Sun, J.-K.; Liu, C.-H.; Liu, C.-Y.; Zhao, X.-M.; Chu, C.-H.; Liu, R.-H. Org. Lett. 2014, 16, 350.

(d) Takaya, H.; Ito, M.; Murahashi, S.-I. J. Am. Chem. Soc. 2009, 131, 10824.

(e) Li, Y.; Liu, B.; Li, H.-B.; Wang, Q.; Li, J.-H. Chem. Commun. 2015, 51, 1024.

(f) Cheng, P.; Wang, W.; Wang, L.; Zeng, J.-G.; Reiser, O.; Liang, Y. Tetrahedron Lett. 2019, 60, 1408.

[3] (a) Kumagai, N.; Matsunaga, S.; Shibasaki, M. J. Am. Chem. Soc. 2004, 126, 13632.

(b) Zhang, J.-L.; Liu, Y.; Song, R.-J.; Jiang, G.-F.; Li, J.-H. Synlett 2014, 25, 1031.

(c) Chakraborty, S.; Patel, Y.-J.; Krause, J.-A.; Guan, H. Angew. Chem., Int. Ed. 2013, 52, 7523.

(d) Pan, C.-D.; Zhang, H.-L.; Zhu, C.-J. Org. Biomol. Chem. 2015, 13,361 .

(e) Hu, M.; Zou, H.-X.; Song, R.-J.; Xiang, J.-N.; Li, J.-H. Org. Lett. 2016, 18, 6460.

(f) Evans, M.-E.; Li, T.; Jones, W.-D. J. Am. Chem. Soc. 2010, 132, 16278 . (g) Chen, Z.; Zhou, Q.; Chen, Q.-N.; Chen, P.; Xiong, B.-Q.; Liang, Y.; Tang, K.-W.; Xie, J.; Liu, Y. Org. Biomol. Chem. 2020, 18, 8677.

[4] (a) Wu, T.; Mu, X.; Liu, G.-S. Angew. Chem., Int. Ed. 2011, 50, 12578 .

(b) Li, X.-Q.; Xu, J.; Gao, Y.-Z.; Fang, H.; Tang, G.; Zhao, Y.-F. J. Org. Chem. 2015, 80, 2621.

(c) Liu, Y.-B.; Yang, K.; Ge, H.-B. Chem. Sci. 2016, 7, 2804.

(d) Chatalova-Sazepin, C.; Wang, Q.; Sammis, G.-M.; Zhu, J. Angew. Chem., Int. Ed. 2015, 54, 5443.

(e) Bunescu, A.; Wang, Q.; Zhu, J.-P. Org. Lett. 2015, 17, 1890.

(f) Bunescu, A.; Ha, T.-M.; Wang, Q.; Zhu, J.-P. Angew. Chem., Int. Ed. 2017, 56, 10555 .

(g) Liu, Y.-Y.; Yang, X.-H.; Song, R.-J.; Luo, S.-L.; Li, J.-H. Nat. Commun. 2017, 8, 14720.

[5] (a) Boivin, J.; Fouquet, E.; Zard, S.-Z.; J. Am. Chem. Soc. 1991, 113,1055

(b) Nishimura, T.; Yoshinaka, T.; Nishiguchi, Y.; Maeda, Y.; Uemura, S. Org. Lett. 2005, 7, 2425.

(c) Nishimura, T.; Uemura, S. J. Am. Chem. Soc. 2000, 122, 12049.

(d) Yang, H.-B.; Selander, N. Chem.-Eur. J. 2017, 23, 1779.

(e) Yang, H.-B.; Pathipati, S.-R.; Selander, N. ACS Catal. 2017, 7, 8441 .

(f) Morcillo, S.-P. Angew. Chem., Int. Ed. 2019, 58, 14044.

(g) Xiao, T.; Huang, H.; An, D.; Zhou, L. Synthesis 2020, 52, 1585.

(h) Song, C.-H.; Shen, X. Yu, F. He, Y.-P.; Yu, S.-Y. Chin. J. Org. Chem. 2020, 40, 3748 (in Chinese).

(宋常华, 沈许, 于芳, 何宇鹏, 俞寿云, 有机化学, 2020, 40, 3748.)

[6] (a) Yu, X.-Y.; Zhao, Q.-Q.; Chen, J.; Chen, J.-P.; Xiao, W.-J. Angew. Chem., Int. Ed. 2018, 57, 15505.

(b) Yu, X.-Y.; Wang, P.-Z.; Yan, D.-M.; Liu, B.; Chen, J.-R.; Xiao, W.-J. Adv. Synth. Catal. 2018, 360, 3601.

(c) He, B.-Q.; Yu, X.-Y.; Wang, P.-Z.; Chen, J.-R.; Xiao, W.-J. Chem. Commun. 2018, 54, 12262.

(d) Lu, B.; Cheng, Y.; Chen, L.-Y.; Chen, J.-R.; Xiao, W.-J. $A C S$. Catal. 2019, 9, 8159 .

(e) Yu, X.-Y.; Chen, J.-R.; Wang, P.-Z.; Yang, M.-N.; Liang, D.; Xiao, W.-J. Angew. Chem., Int. Ed. 2018, 57, 738.

(f) Xiao, W.; Xiao, W.-J. Chin. Chem. Lett. 2020, 31, 3083.

(g) Yu, X.-Y.; Chen, J.-R.; Xiao, W.-J. Chem. Rev. 2021, 121, 506.

[7] (a) Tang, Y.-Q.; Yang, J.-C.; Wang, L.; Fan, M.-J.; Guo, L.-N. Org. Lett. 2019, 21, 5178.

(b) Wu, J.; Zhang, J.-Y.; Gao, P.; Xu, S.-L.; Guo, L.-N. J. Org. Chem. 2018, 83, 1046.

(c) Zhang, J.-J.; Duan, X.-H.; Wu, Y.; Yang, J.-C. Guo, L.-N. Chem. Sci. 2019, 10, 161 .

(d) Yang, L.; Gao, P.; Duan, X.-H.; Gu, Y.-R.; Guo, L.-N. Org. Lett. 2018, 20, 1034.

(e) He, Y.; Lou, J.; Wu, K.-K.; Wang, H.-M.; Yu, Z.-K. J. Org. Chem. 2019, 84, 2178.

(f) Yin, Z.-P.; Rabeah, J.; Brückner, A.; Wu, X.-F. ACS Catal. 2018, $8,10926$.

(g) Yu, J.-X.; Teng, F.; Xiang, J.-N.; Deng, W.; Li, J.-H. Org. Lett. 2019, 21, 9434.

(h) Zhou, X.-S.; Cheng, Y.; Chen, J.; Yu, X.-Y.; Xiao, W.-J.; Chen, J.-R. Chem CatChem 2019, 11, 5300.

[8] (a) Chen, Z.; Zhou, Q.; Wang, Q.-L.; Chen, P.; Xiong, B.-Q.; Liang, Y.; Tang, K.-W.; Liu, Y. Adv. Synth. Catal. 2020, 362, 3004.

(b) Zhang, J.; Zhang, X.-F.; Xie, W.-L.; Ye, S.-Q.; Wu, J. Org. Lett. 2019, 21, 4950.

(c) Liu, Y.; Wang, Q.-L.; Chen, Z.; Li, H.; Xiong, B.-Q.; Zhang, P.-L.; Tang, K.-W. Chem. Commun. 2020, 56, 3011.

(d) Zheng, M.; Li, G.-G.; Lu, H.-J. Org. Lett. 2019, 21, 1216.

(e) Yin, Z.-P.; Rabeah, J.; Brückner, A.; Wu, X.-F. Org. Lett. 2019, 21, 1766 .

(f) Lou, J.; He, Y.; Li, Y.-L.; Yu, Z.-K. Adv. Synth. Catal. 2019, 361, 3787 . 
(g) Shen, X.; Zhao, J.-J.; Yu, S.-Y. Org. Lett. 2018, 20, 5523.

(h) Tian, L.; Guo, S.-Q.; Wang, R.; Li, Y.; Tang, C.-L.; Shi, L.-L.; $\mathrm{Fu}, \mathrm{J} .-\mathrm{K}$. Chem. Commun. 2019, 55, 5347.

(i) Zhao, B.-L.; Shi, Z.-Z. Angew. Chem., Int. Ed. 2017, 56, 12727.

(j) Li, L.-Y.; Chen, H.-G.; Mei, M.-J.; Zhou, L. Chem. Commun. 2017, 53, 11544.

(k) Xiao, W.; Wu, J. Chin. Chem. Lett. 2020, 31, 3083.

(1) Min, Q.-Q.; Li, N.; Chen, G.-L.; Liu, F. Org. Chem. Front. 2019, 6,1200 .

[9] (a) Chen, J.-R.; Hu, X.-Q.; Lu, L.-Q.; Xiao, W.-J. Chem. Soc. Rev. 2016, 45, 2044

(b) Xiong, T.; Zhang, Q. Chem. Soc. Rev. 2016, 45, 3069

(c) Wang, P.-Z.; Zhao, Q.-Q.; Xiao, W.-J.; Chen, J.-R. Green Synth. Catal. 2020, $1,42$.

(d) Jiang, H.; Studer, A. CCS Chem. 2019, 1, 38.

[10] (a) Shaaban, S.; Liang, S.; Liu, N.-W.; Manolikakes, G. Org. Biomol. Chem. 2017, 15, 1947.

(b) Rayner, C.-M. Contemp. Org. Synth. 1994, 1, 191.

(c) Meadows, D.-C.; Gervay-Hague, J. Med. Res. Rev. 2006, 26, 793.

(d) Markitanov, Y.-M.; Timoshenko, V.-M.; Shenko, Y.-G. J. Sulfur Chem. 2014, 35, 188.

(e) Fukuda, H.; Frank, F.-J.; Truce, W.-E. J. Org. Chem. 1963, 28, 1420.

(f) Fang, Y.-Y.; Luo, Z.-G.; Xu, X.-M. RSC Adv. 2016, 6, 59661.

(g) Alba, A.-N.-R.; Companyóa, X.; Rios, R. Chem. Soc. Rev. 2010, 39, 2018.

[11] (a) Rao, W.-H.; Zhan, B.-B.; Chen, K.; Ling, P.-X.; Zhang, Z.-Z.; Shi, B.-F. Org. Lett. 2015, 17, 3552.

(b) Cabrera Afonso, M.-J.; Lu, Z.-P.; Kelly, C.-B.; Lang, S.-B.; Dykstra, R.; Gutierrez, O.; Molander, G.-A. Chem. Sci. 2018, 9, 3186.

(c) Song, R.-J.; Liu, Y.; Liu, Y.-Y.; Li, J.-H. J. Org. Chem. 2011, 76, 1001 .

(d) Xie, L.-Y.; Peng, S.; Liu, F.; Chen, G.-R.; Xia, W.; Yu, X.-Y.; Li, W.-F.; Cao, Z.; He, W.-M. Org. Chem. Front. 2018, 5, 2604.

(e) Xiao, F.-H.; Liu, C.; Yuan, S.-S.; Huang, H.-W.; Deng, G.-J. J. Org. Chem. 2018, 83, 10420.

(f) Howell, B.-A.; Daniel, Y.-G. J. Fire Sci. 2018, 36, 518.

[12] (a) Zheng, D.; Wu, J. Sulfur Dioxide Insertion Reactions for Organic Synthesis, Nature Springer, Berlin, 2017.

(b) Liu, J.-D.; Zheng, L.-Y. Adv. Synth. Catal. 2019, 361, 1710.

(c) Ye, S.-Q.; Qiu, G.-Y.-S.; Wu, J. Chem. Commun. 2019, 55, 1013.

(d) Qiu, G.-Y.-S.; Lai, L.-F.; Cheng, J.; Wu, J. Chem. Commun. 2018, 54, 10405.

(e) Qiu, G.-Y.-S.; Zhou, K.-D.; Gao, L.; Wu, J. Org. Chem. Front. 2018, 5, 691 .

(f) Zhu, J.; Yang, W.-C.; Wang, X.-D.; Wu, L. Adv. Synth. Catal. 2018, 360, 386.

(g) Liu, G.; Fan, C.-B.; Wu, J. Org. Biomol. Chem. 2015, 13, 1592.

(h) Ye, S.-Q.; Zhou, K.-D.; Rojsitthisak, P.; Wu, J. Org. Chem. Front. 2020, 7, 14.

[13] (a) Wu, Y.-C.; Jiang, S.-S.; Luo, S.-Z.; Song, R.-J.; Li, J.-H. Chem. Commun. 2019, 55, 8995.

(b) Yang, J.; Liu, Y.-Y.; Song, R.-J.; Peng, Z.-H.; Li, J.-H. Adv. Synth. Catal. 2016, 358, 2286.

(c) Gong, X.-X.; Wang, M.-J.; Ye, S.-Q.; Wu, J. Org. Lett. 2019, 21, 1156.

(d) An, Y.-Y.; Wu, J. Org. Lett. 2017, 19, 6028.

(e) Rostami, A.; Akradi, J. Tetrahedron Lett. 2010, 51, 3501.

(f) Rahimizadeha, M., Rajabzadehb, G., Hossein, S.-M., AliShiri, E. J. Mol. Catal. A 2010, 323, 59.

(g) Su, W.-G. Tetrahedron Lett. 1994, 35, 4955

(h) Liu, Q.-S.; Lv, Y.-F.; Liu, R.-S.; Zhao, X.-H.; Wang, J.-W.; Wei, W. Chin. Chem. Lett. 2021, 32, 136.

(i) He, F.-S.; Yang, M.; Ye, S.-Q.; Wu, J. Chin. Chem. Lett. 2021, 32,461 .
[14] (a) Liu, T.; Zheng, D.-Q.; Li, Z.-H.; Wu, J. Adv. Synth. Catal. 2018, 360,865 .

(b) Chen, H.-J.; Liu, M.-L.; Qiu, G.-Y.-S.; Wu, J. Adv. Synth. Catal. 2019, 361, 146.

(c) Li, Y.-W.; Mao, R.-Y.; Wu, J. Org. Lett. 2017, 19, 4472.

(d) Woolven, H.; González-Rodríguez, C.; Marco, I.; Thompson, A.-L.; Willis, M.-C. Org. Lett. 2011, 13, 4876.

(e) Vedovato, V.; Talbot, E.-P.-A.; Willis, M.-C. Org. Lett. 2018, 20, 5493.

(f) Wang, Y.; Deng, L.-L.; Zhou, J.; Wang, X.-C.; Mei, H.-B.; Han, J.-L.; Pan, Y. Adv. Synth. Catal. 2018, 360, 1060.

(g) Liu, T.; Zhou, W.; Wu, J. Org. Lett. 2017, 19, 6638.

(h) Deeming, A.-S.; Russell, C.-J.; Willis, M.-C. Angew. Chem., Int. Ed. 2016, 55, 747.

(i) Sun, K.; Shi, Z.; Liu, Z.; Luan, B.; Zhu, J. Org. Lett. 2018, 20, 6687.

(j) Zhou, K.-D.; Chen, J.-Q.; Wu, J. Chin. Chem. Lett. 2020, 31, 2996.

[15] (a) Wang, M.; Chen, S.-H.; Jiang, X.-F. Org. Lett. 2017, 19, 4916. (b) Gong, X.-X.; Li, X.-F.; Xie, W.-L.; Wu, J.; Ye, S.-Q. Org. Chem. Front. 2019, 6, 1863.

[16] (a) Kumar, S.; Verma, S.; Jain, S.-L.; Sain, B. Tetrahedron Lett. 2011, 52, 3393.

(b) Ye, S.-Q.; Li, Y.-W.; Wu, J.; Li, Z.-M. Chem. Commun. 2019, $55,2489$.

(c) Chen, S.-H.; Li, Y.-P.; Wang, M.; Jiang, X.-F. Green Chem. 2020, 22, 322.

(d) Li, Y.-W.; Liu, T.; Qiu, G.-Y.-S.; Wu, J. Adv. Synth. Catal. 2019, 361,1154 .

(e) Gong, X.-X.; Chen, J.-H.; Lai, L.-F.; Cheng, J.; Sun, J.-T.; Wu, J. Chem. Commun. 2018, 54, 11172.

[17] (a) Liu, Y.; Chen, Z.; Wang, Q.-L.; Chen, P.; Xie, J.; Xiong, B.-Q.; Zhang, P.-L.; Tang, K.-W. J. Org. Chem. 2020, 85, 2385.

(b) Xie, X.-Y.; Li, P.-H.; Wang, L. Eur. J. Org. Chem. 2019, 221

(c) Liu, Y.; Wang, Q.-L.; Chen, Z.; Chen, P.; Tang, K.-W.; Zhou, Q.; Xie, J. Org. Biomol. Chem. 2019, 17, 10020.

(d) Zhu, J.-W.; Sun, S.; Xia, M.-F.; Gu, N.; Cheng, J. Org. Chem. Front. 2017, 4, 2153.

(e) Zhu, X.-Y.; Li, M.; Han, Y.-P.; Chen, S.; Li, X.-S.; Liang, Y.-M. J. Org. Chem. 2017, 82, 8761.

(f) Xie, X.-Y.; Li, P.-H.; Wang, L. Eur. J. Org. Chem. 2019, 221.

(g) Zhang, P.-B.; Shi, S.-S.; Gao, X.; Han, S.; Lin, J.-M.; Zhao, Y.-F. Org. Biomol. Chem. 2019, 17, 2873.

(h) Zhang, H.-L.; Li, W.-P.; Zhu, C.-J. J. Org. Chem. 2017, 82, 2199.

(i) Chen, S.; Zhang, P.-B.; Shu, W.-Y.; Gao, Y.-Z.; Tang, G.; Zhao, Y.-F. Org. Lett. 2016, 18, 5712.

[18] (a) Xuan, J.; Xiao, W.-J. Angew. Chem., Int. Ed. 2012, 51, 6828. (b) Ouyang, X.-H.; Cheng, J.; Li, J.-H. Chem. Commun. 2018, 54 , 8745.

(c) Hari, D. P.; König, B. Angew. Chem., Int. Ed. 2013, 52, 4734.

(d) Hari, D. P.; Schroll, P.; König, B. J. Am. Chem. Soc. 2012, 134, 2958.

(e) Ding, W.; Lu, L.-Q.; Zhou, Q.-Q.; Wei, Y.; Chen, J.-R.; Xiao, W.-J. J. Am. Chem. Soc. 2017, 139, 63.

(f) Zhang, M.; Li, N.; Tao, X.-Y.; Ruzi, R.; Yu, S.-Y.; Zhu, C.-J. Chem. Commun. 2017, 53, 10228.

(g) Jiang, H.; Cheng, Y.; Wang, R.; Zhang, Y.; Yu, S. Chem. Commun. 2014, 50, 6164.

(h) Liu, R.-S.; Liu, Q.-S.; Meng, H.-R.; Ding, H.-Y.; Hao, J.-D.; Ji, Z.-Y.; Yue, H.-L.; Wei, W. Org. Chem. Front. 2021, 8, 1970.

[19] (a) Yong, X.; Han, Y.-F.; Li, Y.; Song, R.-J.; Li, J.-H. Chem. Commun. 2018, 54, 12816.

(b) Qin, Q.; Ren, D.; Yu, S. Org. Biomol. Chem. 2015, 13, 10295.

(c) Sun, X.; Yu, S. Chem. Commun. 2016, 52, 10898.

(d) Ouyang, X.-H.; Li, Y.; Song, R.-J.; Li, J.-H. Org. Lett. 2018, 20 , 6659.

(e) Han, Y.-Y.; Wang, H.; Yu, S. Org. Chem. Front. 2016, 3, 953. 
(f) Sun, J.; He, Y.; An, X.-D.; Zhang, X.; Yu, L.; Yu, S. Org. Chem. Front. 2018, 5, 977.

[20] (a) Li, Y.; Mou, T.; Lu, L.; Jiang, X. Chem. Commun. 2019, 55, 14299.

(b) Zhou, H.; Deng, X.; Ma, Z.; Zhang, A.; Qin, Q.; Tan, R.-X.; Yu, S. Org. Biomol. Chem. 2016, 14, 6065.

(c) Chen, J.-R.; Hu, X.-Q.; Lu, L.-Q.; Xiao, W.-J. Acc. Chem. Res. 2016, 49, 1911.

(d) Li, M.; Yang, J.; Ouyang, X.-H.; Yang, Y.; Hu, M.; Song, R.-J.; Li, J.-H. J. Org. Chem. 2016, $81,7148$.

(e) Liu, Y.; Wang, Q.-L.; Chen, Z.; Zhou, Q.; Xiong, B.-Q.; Zhang, P.-L.; Tang, K.-W. Chem. Commun. 2019, 55, 12212

(f) Wang, P.-Z.; Gao, Y.; Chen, J.; Huan, X.-D.; Xiao, W.-J.; Chen,
J.-R. ChemRxiv doi: 10.26434/chemrxiv.13325669.v1.

[21] (a) Yan, D.-M.; Chen, J.-R.; Xiao, W.-J. Angew. Chem., Int. Ed. 2019, 58,378

(b) Yan, J.-M.; Cheo, H.-W.; Teo, W. K.; Shi, X. C.; Wu, H.; Idres, S.-B.; Deng, L.-W.; Wu, J. J. Am. Chem. Soc. 2020, 142, 11357.

(c) Huang, B.-B.; Zhao, Y.-T.; Yang, C.; Gao, Y.; Xia, W.-J. Org. Lett. 2017, 19, 3799.

(d) Xuan, J.; Feng, Z.-J.; Duan, S.-W.; Xiao, W.-J. RSC $A d v .2012$, 2,4065 .

(e) Zhang, J.; Wang, L.; Liu, Q.; Yang, Z.; Huang, Y. Chem. Commun. 2013, 49, 11662

(f) Liu, Y.; Wang, Q.-L.; Xiong, B.-Q.; Zhang, P.-L.; Yang, C.-A.; Gong, Y.-X.; Liao, J.; Zhou, Q. Synlett 2018, 29, 2396.

(Cheng, F.) 